\title{
Réflexions sur le langage d'un relief de 1512 : ni Renaissance ni gothique ou comment dépasser les limites de l'histoire de l'art
}

Mots clés: cheminée de l’ancien palais épiscopal de Ljubljana, Renaissance, gothique, hybridation, langage, langage artistique, art et linguistique

Les restes des reliefs d'une cheminée de l'ancien palais épiscopal de Ljubljana, capitale de la Carniole, sont l'une des œuvres les plus étranges au début du XVI $\mathrm{XI}^{\mathrm{e}}$ siècle (illustration 21). Anonymes, mélangeant motifs floraux et figures fantastiques, à quels contextes faut-il les rattacher vers 1512 ? C'est une question en exergue essentielle, qui doit précéder toute introduction générale, toute description, on risque sinon de se laisser emporter par les méthodes classiques de l'histoire de l'art et de passer à côté de leur métasignification - tant pour les hommes de 1512 que pour nous.

Car, disons-le d'emblée, l'écueil le plus dangereux est d’appliquer "notre" histoire de l'art à des protagonistes que nous ne connaissons pas, l'autre étant de se contenter d'une description "objective" pour éviter de s'empêtrer dans des interprétations qui seraient trop mal étayées. Nous voulons montrer que les historiens de l'art naviguent souvent entre Charybde (appliquer "notre" histoire) et Scylla (description "objective") en fuyant les problématiques plutôt que de les affronter.

\section{Etat de la question}

Ces reliefs sont l'un des rares restes du premier palais épiscopal construit en 1511 et seuls deux historiens de l'art ont affronté cette construction, Nace Šumi et Emilijan Cevc. Les intuitions du premier ont été confirmées avec le temps, il voit dans ce palais une œuvre où l'on ressent une sensibilité aux proportions adoptées dans la péninsule italienne (Šumi, 1966, 36). Lapparent manque de signes extérieurs de richesse serait plus la marque d'un goût "élégant", ce qui est affirmé par la plaque encore visible aujourd'hui. Nous n'allons pas retranscrire toute l'inscription, très sobre en lettres latines classiques, surmontée de deux dauphins et de l'ours du blason de la famille Raubar, mais le début et la fin sont très révélateurs : 
D.O.M. / CHRISTOPHORUS / RAUBAR (...) SVI. VETVSTAR.AEDIVM / ANGVSTIAM.AC.DEFOR / MITATEM.PEROSVS.A / FVNDAMENTVS. DIRV/TAS.IN.HANC.NOVAM / FACIEM. PVBLICAE / ELEGANTIAE. AC.PRIVA./TAE.COMMODITATI / CONSVLENS.SIBI / SVIQ. SVCCESS / RESTAVRAVIT / BONISQ.VIRIS.OMNIBVS. / PERPE/TVO/PRIVILEGIO. PATERE. / VOLVIT / M D X II.

Le choix de cette écriture et le mot élégant doivent nous renvoyer à Laurentius Valla, Lorenzo de Vale (1407-1457), à ses Elegantiae linguae Latinae (1435-1444; publié à titre posthume en 1471 et réimpriméa six fois jusqu’en 1536).

Les initiales en exergue signifient Deo Optimo Maximo, qui reprend le Iovi Optimo Maximo romain. Remarquons que ce n'est pas seulement une adaptation "chrétienne" où Dieu remplacerait Jupiter, plus profondément cela renvoie aux dissensions internes des humanistes et ici c'est plus subtilement l'Antiquité chrétienne qui se réapproprie l'Antiquité gréco-romaine. Ce n’est donc pas un christianisme qui se place au-dessus du paganisme, c'est un christianisme "humaniste" qui se place au-dessus du christianisme "scolastique" (et donc peut prétendre en intégrer une partie), dans cette relation triangulaire il est dangereux d'affirmer que les humanistes admirent le paganisme ou renient la scolastique.

Šumi, cependant, ne considère que l'architecture extérieure. Le second, Emilijan Cevc, n’approfondit que l'analyse des reliefs de la cheminée, voilà ce qu'il en dit :

le palais ... était en son temps orné par une cheminée, dont le cadre décoré en pierre Renaissance a été déplacé de la cour (...) au premier étage. (...) ici s'alignent des êtres mythologiques fantastiques dont les corps se terminent en queues de serpents et de dauphins, entre eux il y a des tritons et des sphinges. La plupart des figures sortent des représentations $d u$ monde antique, certaines sont aussi issues des livres médiévaux tardifs de sciences naturelles et de fables. Cependant, les monstres marins en particulier - "aquatici monstriculi" - étaient très appréciés dans l'inventaire décoratif de la Renaissance. Des têtes de boucs animent les angles des chapiteaux et deux consoles à volute se déroulent sous le linteau. - Renaissance ? Oui, mais il serait difficile de dire exactement s'il s'agit d'un sculpteur du Nord de l'Italie ou d'Europe centrale. (...) Il est manifeste que le sculpteur de Ljubljana s'est servi de modèles graphiques tels qu'on en rencontre en bordure des textes imprimés à Venise aux $X V^{e}$ et $X V I^{e}$ siècles, il n'est pas exclu qu'on pourrait les rechercher aussi en direction de la Lombardie $(\text { Milan })^{1}($ Cevc, 1981, 26).

1 »... je svoj čas krasil kamin, katerega renesančno oblikovani kamnitni okvir so zadnja leta prenesli z dvorišča (...) v prvem nastropju. (...) tu se vrste fantastična mitološka bitja, ki jim prehajajo telesa $\mathrm{v}$ repe kač in delfinov, vmes so tritoni in sfinga. Večina figur je nabrana iz antičnega predstavnega sveta, 
Alors, après Cevc, posons-nous la question : - Renaissance ? Il répond «oui » mais nous retenons que le mot qui suit est «mais » et en rhétorique on sait qu'un " oui mais" est un "non". Si nous revenons à notre question du début "à quels contextes faut-il rattacher cette œuvre? », relevons le vocabulaire employé par Cevc: «Renaissance, fantastique, mythologique, antique, médiéval tardif, sciences naturelles, fable, Nord de l'Italie, Europe centrale, modèle graphique, texte imprimé ». N'accusons pas à tort Cevc d’être indécis ou peu précis, lui-même est mal à l'aise et prudent avec la "Renaissance", ce n'est pas pour rien que son livre s'intitule " Entre le gothique et le baroque ». Le reste est une suite de termes généraux qui sopposent, il est alors difficile d'oublier un quelconque référent possible, n'y manque que la tradition orale, seul langage qui n’est pas fixé sur un support qui permet de rendre l'hypothèse vérifiable.

Relevons aussi une autre phrase : « le sculpteur de Ljubljana sest servi de modèles graphiques ». D’abord on ne sait rien sur la "nationalité" ou l'origine du sculpteur (mais le terme latin de natio était déjà couramment utilisé à lépoque, dans un sens bien différent de celui que nous lui attribuons aujourd'hui). Ensuite, d'où viennent les modèles (s'il y en avait ! pourquoi ne pas imaginer qu'ils ont été dessinés pour loccasion ?) : est-ce le commanditaire qui les a proposés ? Le sculpteur les avait-il déjà ? In fine, comment les a-t-il utilisés?

Avant de répondre à ces questions, nous pouvons ajouter quelques mots sur les personnalités probablement liées de près ou de loin à cette cheminée. Christophe Raubar en est le commanditaire, c'est une évidence, son blason est au milieu du linteau. Ses contemporains ne s'y trompaient pas lorsqu'ils le considéraient comme l'esprit le plus brillant de Carniole, de Styrie et de Carinthie : "Tu enim imprimis inter Illiricas nationes fulgentissimo quodam splendore collucescis (...) ut in Stiria, Carinthia, Carniola tam infimates quam summates te diligant. "(Simoniti, 1979, 76). Raubar entretenait une cour de 90 personnes, rassemblant artistes et intellectuels. Il est bien sûr très frustrant de ne presque rien savoir sur cette cour, on devine qu'il est essentiel de mieux la connaitre si l'on veut comprendre l'histoire de la région. ${ }^{2}$

Son père Nicolas était gouverneur de Trieste, Christophe Raubar n'a que douze ans lorsque l'évêché de Ljubljana lui est promis, c'est-à-dire en $1488 .{ }^{3}$ Il fait ses études

nekatere pa so prevzete tudi iz poznosrednjeveških naravoslovnih knjig in basni. Sicer pa so bile zlasti morske pošasti - « aquatici monstriculi » - v renesančnem okrasnem inventarju zelo priljubljene.

Ogle kapitelov poživljajo ovnove glave, pod gredo se uvijata volutni konzoli. - Renesansa? Da, a težko bi bilo določno povedati, ali gre za severnoitalijanskega ali srednjeevropskega klesarja. (...) Kazno je, da se je ljubljanski klesar oprl na grafične predloge, kakršne srečamo kot bordure v beneških tiskih 15. in 16. stoletja, ni pa izključeno, da bi smeli iskati tudi v lombardski (milanski) smeri.«

2 Nous entendons par là qu'il n'y a pas de travaux publiés, mais il existe un riche fonds aux Archives archiépiscopales de Ljubljana (communication orale de Lilijana Žnidaršič Golec, archiviste aux Archives nationales de Slovénie).

3 En recoupant tous les témoignages et les informations disponibles, Simoniti prouve que le futur 
à Padoue, de droit canonique et de droit civil, terminées en 1501. Dans une mission à Rome, en 1504, il impressionne le pape Jules II par son talent oratoire. En 1505, il intervient auprès de plusieurs princes au nom de l'empereur Maximilien $\mathrm{I}^{\text {er }}$; en 1506, il retourne à Rome et va à Naples pour rencontrer Ferdinand d'Aragon, où il reste jusqu’en 1507. L'année suivante, il rencontre l'impératrice à Constance.

Cette même année, par décret de Maximilien $\mathrm{I}^{\mathrm{er}}$, Raubar est nommé abbé commendataire du couvent bénédictin d’Admont, le 8 février 1508 ; le même mois, il est évêque de Ljubljana. Pour protéger ses Etats pontificaux, le pape Jules II réunit la ligue de Cambrai avec Louis XII d'Orléans, Ferdinand d'Aragon et Maximilien I ${ }^{\text {er }}$ de Habsbourg ; cette ligue dirigée contre Venise est officialisée le 10 décembre 1508. Raubar est aussitôt nommé commissaire de l'armée ; connu de l'empereur et du pape, il n'est pas étonnant qu'un évêque soit promu à de telles fonctions. Le 20 avril 1509, il est coadjuteur et administrateur du couvent bénédictin de Seckau, également en Styrie ; sous Raubar, ce couvent devient siège d'un évêché. En 1510, il devient membre du tribunal supérieur de Styrie. En 1515, il participe au congrès de Vienne avec le titre de maréchal des armées. A côté de cette activité, signalons enfin que le couvent d’Admont possède une riche bibliothèque, ne ménage pas ses moyens pour ses ambitions artistiques, entretient deux palais à Graz et à Salzbourg (List, 1974).

Aux côtés de l'évêque, se trouve la personnalité peut-être la plus intéressante de lépoque, Augustin Tyfernus (Simoniti, 1979, 83-112; Šumi, 1966, 45-47), pseudonyme d'Augustin Prygl, ${ }^{4}$ né à Laško vers 1475 . Avec Raubar, il fait ses études à l'université de Padoue. Il l'accompagne sûrement à Rome en 1504.

Tyfernus est d'abord connu par ses contemporains comme collectionneur d'inscriptions romaines, parfois juste sous le nom d'Antiquus Austriacus. On sait aussi que Tyfernus était à Naples en 1507 (donc avec Raubar), où il a rencontré fra Giovanni Giocondo da Verona (1435-1515), l'un des grands précurseurs de l'épigraphie romaine (illustration 1). Tyfernus mène ses propres recherches en Italie, collecte les travaux précédents, et fait publier chez Sigismund Maier, imprimeur allemand installé à Naples, un recueil de 52 feuillets intitulé Libellus de mirabilibus civitatis Puteolorum et locorum vicinorum ac de nominibus virtutibusque balneorum ibidem existentium. ${ }^{5}$

Lautre facette de Tyfernus est son activité d'architecte, ou plus modestement " preposito a la fabrica del palazo ", tel que l'écrit Augustino Bonomo à son neveu Giovanni Battista, à Trieste, en 1519, faisant allusion au palais épiscopal de Ljubljana.

évêque est bien né autour de 1476 ; sur sa biographie complète (Simoniti, 1979, 62-69).

4 Laško, sa ville natale, est Tüffer en allemand, doù son pseudonyme latin; on trouve aussi les orthographes Tiernvs, Tifernus, Tiffernus, Prigl, Prugel, Prugl et Tyfernus-Prygl.

5 Ce texte est encore réimprimé en 1723, avec des commentaires, parmi d’autres textes sur l’Antiquité. 
Sa première réalisation connue est le palais épiscopal de Vienne (détruit depuis), commandé par lévêque Georg Slatkonia (1456-1522), alors chancelier, dans la première décennie 1500/1510. Relevons que Georg Slatkonia, évêque de Vienne à partir de 1513, est né à Ljubljana ; il est surtout connu comme musicien et compositeur. En raison du tremblement de terre de 1511, lévêque Raubar confie à Prygl la reconstruction de la résidence épiscopale de Gornji Grad.

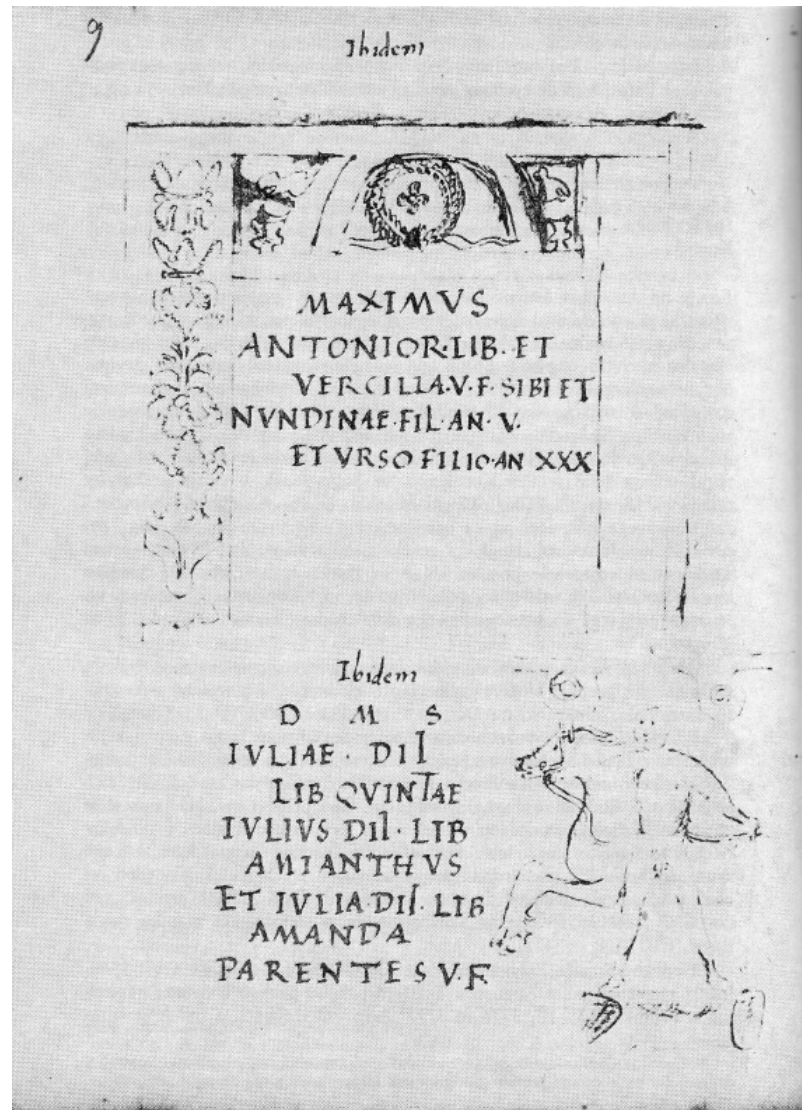

illustration 1 : Augustin Prygl, page manuscrite source : Simoniti, 1979, 92

Quant à celui de Ljubljana, il a subi d'importantes modifications. Ce dernier dut être rasé après le tremblement de terre de 1511, la première pierre est posée le 4 mai 1512. ${ }^{6}$ Aux yeux des contemporains, les nouveautés qu'il introduit sont les proportions du bâtiment, c'est vrai qu'il y a une modeste rupture avec la tradition locale (Šumi, 1966, 36). 
Pour notre propos, il faut encore évoquer le Styrien Bernard Perger, proche du cercle de Raubar et peu de temps auparavant recteur de l'université de Vienne. Il se fait connaître en rédigeant sa propre méthode d'enseignement du latin, la Grammatica nova. Cette grammaire, publiée à Venise en 1479, est loin dêtre un manuel anodin supplémentaire, elle est à l'origine d'un violent conflit. En cela, la polémique viennoise est contemporaine du Hollandais Alexander Hegius ( $† 1498)$ qui publie vers 1480, à Deventer, ses Invectives contre les méthodes de l'enseignement scolastique et son latin "barbare". Les humanistes préconisent un enseignement moins formellement centré sur la grammaire et plus porté vers l'élégance de l'expression, écrite et orale (Simoniti, 1979, 154-174). Ce tour d'horizon serait incomplet sans le Vénitien Francesco Colonna (1433-1527), auteur de l'Hypnerotomachia Poliphili; le livre a eu un énorme succès en 1499, précisément lorsque Raubar et Prygl étudient à Padoue, et nous allons souvent le convoquer. ${ }^{7}$

\section{Préambule : y avait-il des styles vers 1512 ?}

Dans le but d'évacuer de notre esprit certaines idées tenaces qui polluent notre regard sur le passé, faisons un peu le ménage. L'une des présuppositions à chacune de nos réflexions est celle du style lorsque nous cherchons à situer une œuvre. Il n'est donc pas vain de faire une petite mise au point sur la Renaissance et le gothique, termes utilisés par Cevc, un bref survol est très révélateur.

Le mot gothique vient du bas latin gothicus, "relatif aux Goths » et au XV siècle on en dérive l'adverbe gothique pour qualifier une façon d'écrire, notamment chez Lorenzo Valla dans le sixième livre de son Elegantiae linguae Latinae. ${ }^{8}$ Le mot est presque aussitôt utilisé en français, en 1482, dans son sens historique (donc strictement relatif au peuple antique des Goths). Relatif à l'art, on le trouve pour la première fois sous la plume de Raphaël en 1519, dans une lettre qu'il écrit au pape Léon X ; le terme est péjoratif, synonyme de "barbare". Une légende tenace veut que ce soit Vasari, dans les Vite en 1550, qui ait le premier utilisé le terme gotico dans un texte imprimé et largement diffusé ; en réalité il utilise le terme todesco, allemand, traduit en latin gothieus par un jésuite français en 1610. Vasari n’a jamais utilisé le mot gothique... Il parle certes du peuple des Goths, dans le sens de barbares, et il est vrai qu'il oppose Antiquité et Allemands dans la phrase «gli ordini antichi buoni e non la todesca e barbara» (Vasari, 1550, 298), mais les Goths peuvent être mis en apposition avec les Grecs pour simplement désigner une période antique aux apports multiples.

$7 \quad$ Paru chez Alde Manuce à Venise ; le texte original avec les illustrations est en accès libre sur le site de Gallica, http://gallica.bnf.fr/ark:/12148/bpt6k587910.r=francesco+colonna.langFR.

8 Il y a la célèbre phrase de Valla « Tout ce qui est gothique est mauvais, et tout ce qui est mauvais est gothique ", mais nous n'avons trouvé aucune référence précise, il s'agit alors probablement d'une légende tenace basée sur une mauvaise traduction (telle la méprise Vasari/gothique). Remarquons que le style architectural que nous qualifions de gothique se disait alors «style français ». 
Le terme passe en français, dès 1615 il est employé pour désigner une période artistique, il apparaît dans ce sens en allemand seulement à la fin du XVIII siècle, notamment chez Goethe dans Von Deutscher Baukunst en 1773. Ce n'est qu'au XIX siècle que les autres langues adoptent le terme, comme dérivé du français ; aussi étonnant que cela paraisse, il n'apparaît dans son sens contemporain qu'en $1824 .^{9}$

Quant à la Renaissance, Stendhal parle de « renaissance de la peinture » en Italie en 1817. Cependant, le terme n’apparaît qu’en 1828 pour désigner une période historique littéraire, en 1829 il désigne une période historique artistique, en français, il essaime ensuite dans les autres langues. Il faut attendre Michelet en 1840-41 pour qu'il parle de Renaissance comme période de la Renaissance de l'homme et de la culture. ${ }^{10}$ Febvre démontre que le terme est éthique, non historique, et correspond à un moment donné de la vie intérieure de Michelet. Il est difficile de soutenir que le terme cherche alors à définir une réalité historique.

S’ensuivent plusieurs remarques pour notre cheminée :

- ni les termes de Renaissance ni de gothique n'existaient pour qualifier un style ou une période artistique, ces notions étaient étrangères aux artistes vers 1512.

- le mot "elegantiae " dans l'inscription du palais épiscopal, et lécriture choisie, renvoient indubitablement à Lorenzo Valla, qui écrivait en latin, dans une moindre mesure à Perger qui reprend le combat de Valla. ${ }^{11}$

- en art, le mot gothique prend alors le sens de "barbare", sans connotation géographique ; pour les Grecs anciens, les barbares étaient tous ceux qui ne parlaient pas grec, pour Raphaël et Vasari ce sont tous ceux qui ne créent pas comme eux, cela n'a même aucun rapport avec "l'Italie" opposées aux barbares. Nombre d'artistes nés dans la Péninsule avaient un "style barbare" !

- dans le conflit Perger/Celtis, Celtis parle de "Germains" et de "Celtes" (et non de Goths), en Italie on parle de "Tedeschi” ou d' "ultramontani", Italien était un terme souvent péjoratif en Europe, pour certains intellectuels la "langue italienne" était un ensemble de dialectes "dégénérés" parlés par des gens incultes, incapables de parler le latin. Les intellectuels du Saint Empire romain germanique se sentaient de plus dignes héritiers de l'Empire romain que ces rustres d'Italiens! Celtis est le plus illustre représentant de ce point de vue parmi les humanistes.

9 Cf. "Gothique, adj. et subst." dans le Trésor de la langue française informatisé, http://atilf.atilf.fr/; Jakob Grimm et Wilhelm Grimm éd., " Gotik, f. » et " gotisch, adj. », Deutsches Wörterbuch, t. 8, col. 998-1016 ; Haslag, 1963 ; Holbrook, 1941.

10 Cf. "Renaissance, subst." dans le Trésor de la langue française informatisé, http://atilf.atilf.fr/; Febvre, 1992 (texte établi d’après les cours donnés au Collège de France en 1942-43).

11 Preuve qu'il n'est pas vain d'insister sur ce mot, le traité de Valla est traduit en français en 1507 sous le titre Les Elégances. 
$\mathrm{Au}$ vu de ces éléments, la seule conclusion qui s'impose est que les intellectuels autour de Raubar ne se préoccupaient nullement dêtre "Renaissance" ou "gothique", notions absurdes appliquées à ce contexte, ils veulent juste marquer qu'ils ne sont pas "barbares". Cela nous rappelle cette réponse d'Ange Politien (1454-1494) à Ermolao Barbaro (1454-1493) qui veut aider le maître : « si dix Barbaro métaient donnés, j'aurais bon espoir que sous peu les lettres grecques et latines soient sauvées de la barbarie $» .^{12}$ Mais, à Ljubljana, que signifie ne pas être barbare ? C'est parler un langage porteur de culture, dont on peut trouver des origines nobles sur son propre territoire ; cela n'empêche pas d'accepter l'art médiéval et de considérer la supériorité du christianisme.

Le terme "barbare" est donc une idée clef à l'époque, le mot est abondamment utilisé, mais elle dépend éminemment du point de vue de celui qui l'emploi. Il faut donc chercher dans les reliefs de la cheminée les éléments qui définissent cette culture qui n'est pas "barbare", ou plutôt comment les éléments de cette cheminée définissent le monde "non-barbare" vu de Ljubljana : non, la langue parlée n’est pas celle de Raphaël à Rome, on parle ici une langue qui intègre un fond culturel très élargi. Disons-le d'emblée : ce langage "non-barbare" renvoie à la fois à la grandeur de l'Antiquité romaine et du Saint Empire romain germanique. Les provinces de Carniole, Styrie et Carinthie sont alors bien "barbares" à ce moment, nous en avons deux témoignages. Le premier en 1444, lorsque le futur pape Pie II s'exclame : " nunc in Stiria, nunc in Carinthia, nunc in Carniola inter medios barbaros sevasque nationes constitutus ». ${ }^{13}$ Et vers 1540, le poète polonais Clément Janicki (documenté entre 1516 et 1542/43) traverse la Styrie, au retour de Padoue vers sa terre natale; malade il est recueilli par des paysans "barbares" et laisse ce souvenir dans ses poèmes :

Barbarius nihil est Alpina gente ferumque

(...) Aduena despectus cunctis, est hospes ut hostis

Itala praecipue pallia quisquis habet. ${ }^{14}$

En fait, il reprend Ovide, dans cet étonnant renversement de perspective (Ovide, Triste, v. 35-37) :

Exercent illi sociae commercia linguae :

Per gestum res est significanda mihi.

Barbarus hic ego sum, qui non intellegor ulli.

12 Cité par Nicolas Petit, professeur poète, humaniste et régent ès arts au collège de Montaigu, dans la préface de ses Silvae, recueil de poésies de 3000 vers environ, publié en 1522, chez Jean de Gourmont.

13 Dans des lettres de 1444 et de 1445, il a des termes similaires pour la cour de l’empereur Frédéric III en Autriche et pour la ville de Bâle (Simoniti, 1979, 16, 18).

14 Les textes de Janicki sont édités par Ludwik Cwiklinski, Carmina, Universitatis Iagellonicae, Cracovie 1930 ; l'idée de "barbare" chez Janicki et d’autres humanistes est analysée chez Descoings, 2007. 


\section{Que voyons-nous? Ou du danger de nommer ce qui est inconnu}

Evitons donc de porter le moindre jugement sur cette cheminée, ne faisons pour l'instant aucune supposition. Contentons-nous d'une simple description. Et là déjà nous sommes face à un problème insurmontable : comment nommer les motifs, les êtres que nous voyons? Nous ne devons pas négliger ce problème sémantique car toute énonciation d'un nom fait appel à un fonds culturel ou linguistique qui guide inconsciemment nos conclusions. La meilleure parade est d'utiliser le vocabulaire de l'époque, mais où le retrouver ? Et quand on le retrouve, dans quelle langue étaitil ? Etait-ce une traduction, une adaptation de termes anciens ? Que signifiait alors traduction? Signalons que le mot "traduction" n'existait même pas vers 1512, on parlait alors de «translatio » en latin... ${ }^{15}$

Ces questions ne sont pas absconses et propres à l'histoire de l'art contemporaine, elles étaient d'une actualité brûlante pendant des siècles. Que l'on songe à la querelle des universaux, dont un des aspects est nommé nominalisme à la fin du XV siècle, le débat s'envenime à tel point qu'on a défendu d'en parler et que cette interdiction n'a été levée à Paris qu'en 1481. Dans cette querelle, on oppose sententia vacum (doctrine des mots : les idées générales sont des flux de sons, pas plus) et sententia rerum (doctrine des choses : les idées générales expriment les réalités), on différencie le genre, l'espèce et la qualité. Rapporté à notre cheminée - sachant quautour de Raubar on connaissait ces questions - on peut s'interroger sur le degré de réalité et le sens qu’on attribuait à ces figures : car leur donner un nom, c'est leur conférer une existence dans l'esprit du locuteur mais cela n'induit pas leur réalité en dehors de cette énonciation. Ces figures ne sont que mots, aujourd'hui nous ne savons pas dans quelle dimension il faut les situer, c'est pour cela qu'il est essentiel dêtre attentif et prudent sur ce point, celui du problème de la relation entre ces entités linguistiques et les représentations mentales. ${ }^{16}$

Par son vocabulaire, «tritons, sphinge », Cevc renvoie à l'Antiquité gréco-romaine mais peut-on réellement savoir comment l'artiste et le commanditaire les ont nommés? Quant à l'expression " aquatici monstriculi », c'est plus un terme descriptif à connotation médiévale ou antique, mais ce n'est pas un nom. C’est cependant manquer de beaucoup de prudence. Ce problème est loin dêtre anecdotique car avant même de chercher un nom, il y a cette question encore plus profonde : de quels êtres s'agit-il : deêtres étranges?

15 Sur cette question essentielle, le cas précis de la Bourgogne au XVe siècle est très éloquent in Doudet, 2007.

16 Plusieurs penseurs se sont illustrés sur ces questions posées par Aristote, souvent polémiques, dès le $\mathrm{XI}^{\mathrm{e}}$ siècle, débattues dans toute l'Europe. Le débat gagne Vienne avec Jean Buridan qui s'y exile, au milieu du XIVe siècle, Guillaume d'Ockham ayant ravivé les braises. La bibliographie est fournie, on peut se reporter à Klima, 2008 ; Biard, 2009. 
d'êtres imaginaires ? de croyances ? de simples reproductions de modèles anciens ? de créations ludiques? de personnages oniriques $?{ }^{17}$ Il est très révélateur que les chercheurs qui s'occupent de l'hybridation et de la métamorphose ont renoncé à donner des noms aux créatures, même pour une simple description, ils préfèrent les classer en fonction de critères comme anthropomorphe/zoomorphe, le procédé mis en œuvre, l'utilisation d'éléments récurrents (pelage, plumes, griffes, sabots, serpentiforme, ailes, tête, serres, membres, objet, etc. ; Thénard-Duvivier, 2009). Les chercheurs sont devenus conscients que nommer ces êtres les arrache à leur contexte.

Si on utilise leurs recommandations les plus récentes, cela donne :

- Sur le linteau de Ljubljana, le premier être est donc un être zoomorphe serpentiforme ailé à tête qui tient du reptile et de l'oiseau (illustration 2) ; il est difficile de dire s'il est recouvert d'écailles ou de plumes, si ses membres supérieurs sont terminés par des serres ou des griffes, si son museau est un bec ou non. Il est impossible dêtre plus précis, tout terme qualificatif est une interprétation et non une description. Ce qui narrange pas nos affaires, c'est que nous ne savons pas dans quelle langue le commanditaire et le sculpteur ont discuté : en allemand, en italien, en latin, en slovène ? Car chacune de ces langues proposent d'autres possibilités pour créer ces êtres hybrides, il n'est pas sûr que l'un ait vraiment entendu ce que l'autre a dit. Autrement dit, nous ne savons pas si nous voyons les instructions du commanditaire ou l'interprétation du sculpteur. Si nous sommes dans le second cas, nous ne saurons jamais qu’est-ce qui est représenté, l'“original” aurait été effacé par une exécution maladroite ou une mauvaise compréhension des idées et mots employés.

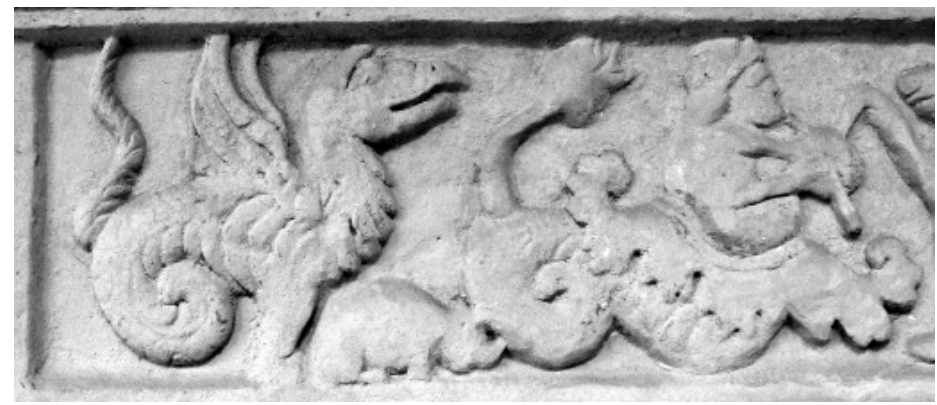

illustration 2 : Anonyme, cheminée du palais épiscopal de Ljubljana, détail, c. 1512 source : photo D. Krašovec

- Suit ce qui semble être : un pourceau ? Pourquoi pas un rhinocéros? Une tortue? Un fourmilier? (illustration 2)

17 Dans le récit de Colonna, l'histoire du héros Poliphile est celle d'un rêve dans un rêve ; nous ne prétendons pas que les figures hybrides sont oniriques, mais on ne peut exclure cette dimension. 
- Puis un être anthropomorphe musicien et coiffé d'un chapeau, serpentiforme avec une queue de bovin, avec une étrange "robe" de tissu ? En feuille d'acanthe ? (illustration 2) On sait seulement qu'il ne s'agit ni d'un centaure, ni d'un léocentaure, bien qu'il y ait parenté. Son instrument ressemble à une cornemuse mais beaucoup d'instruments à lépoque fonctionnaient sur ce principe.

- Suit un ours dressé (forme domestique d’un animal sauvage) (illustration 3).

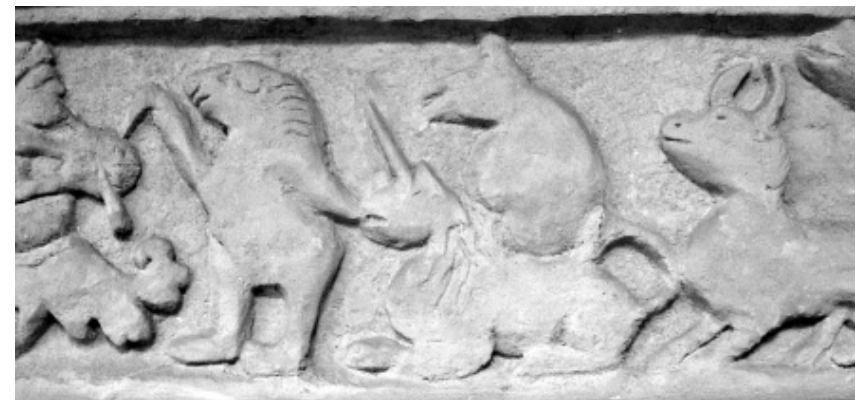

illustration 3 : Anonyme, cheminée du palais épiscopal de Ljubljana, détail, c. 1512 source : photo D. Krašovec

- Puis une licorne supportant ce qui semble être un ours plus petit ou un porc (illustration 3). Vers 1500, le motif de la licorne nest pas étranger à la région ; le Musée régional de Klagenfurt conserve une belle Chasse à la licorne, provenant de l'église paroissiale de Gospa Sveta, en Carinthie slovène..$^{18}$ La licorne ne renvoie néanmoins à aucune sphère culturelle précise, on la trouve déjà chez les Grecs anciens, les Romains, et dans la Bible, en raison d'une erreur de traduction dans la Vulgate.

- Vient un taureau, ou un bœuf, ou une vache (illustration 4).

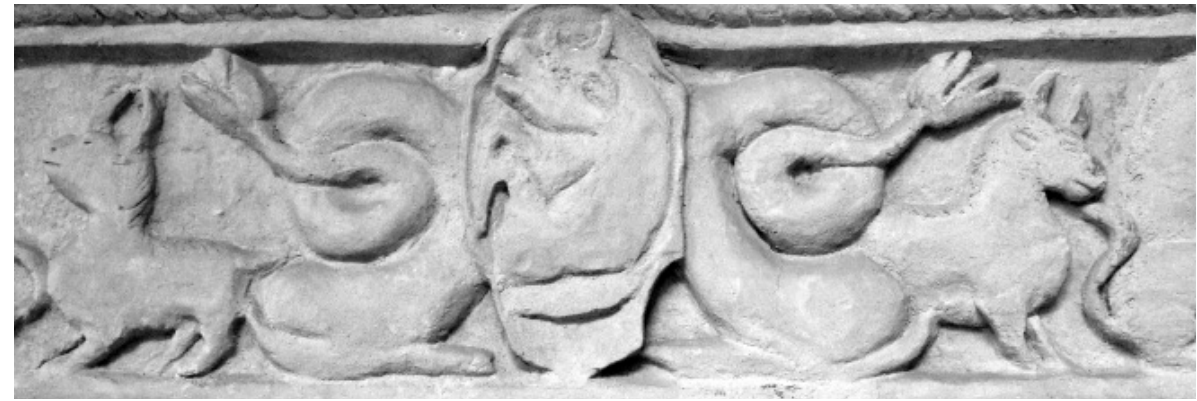

illustration 4 : Anonyme, cheminée du palais épiscopal de Ljubljana, détail, c. 1512 source : photo D. Krašovec

18 Nous ne nous attardons pas sur l'iconographie, dont l'interprétation est spéculative; Cevc en donne une interprétation convaincante (Cevc, 1970, 119-121, 307), en s'appuyant sur plusieurs études autrichiennes et slovènes qu'il cite en note. Sur le motif de la licorne, signalons aussi la thèse de doctorat de Faidutti, 1996. 
- Au centre, il y a le blason des Raubar encadré de deux dauphins (illustration 4).

- Suivent un bovin, un chien debout (?), un lion (?) et un ours debout (?) (illustration 5).

- Un autre être musicien est un semblable du précédent mais celui-ci joue de la flûte ou d'un instrument apparenté (illustration 5).

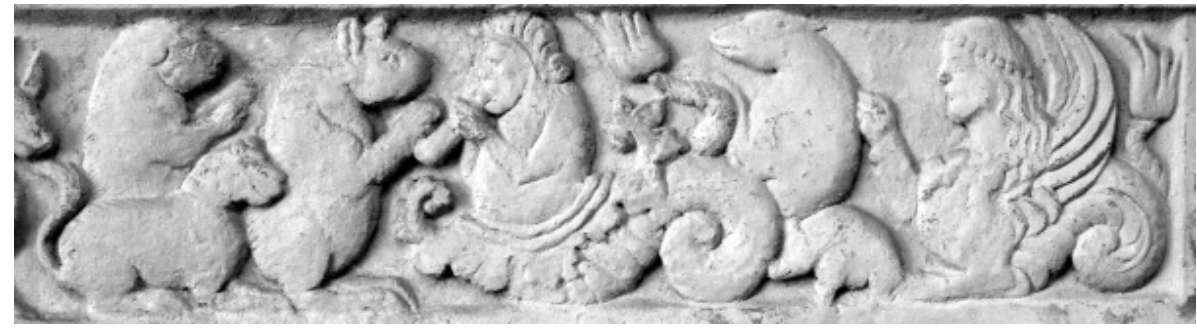

illustration 5 : Anonyme, cheminée du palais épiscopal de Ljubljana, détail, c. 1512 source : photo D. Krašovec

- Suivent deux suidés (une truie, un cochon, un pourceau, un sanglier ?) (illustration 5).

- Enfin, le linteau se termine à droite par un être féminin ailé serpentiforme, avec des griffes ou des serres aux membres postérieurs (illustration 5). Il ne s'agit pas forcément d'un sphinx, ce peut être une harpie, une kère, ou autre chose - ou rien de cela.

- Ce linteau est supporté par deux éléments carrés avec des êtres serpentiformes ailés et des dauphins (illustration 6).

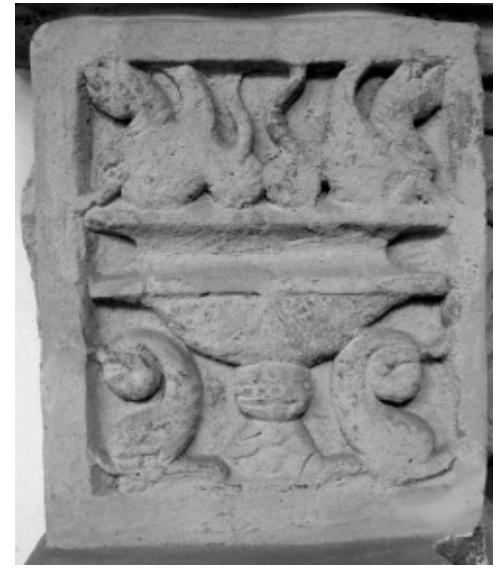

illustration 6 : Anonyme, cheminée du palais épiscopal de Ljubljana, détail, c. 1512 source : photo D. Krašovec 
- Ces éléments sont eux-mêmes supportés par des chapiteaux ayant chacun deux têtes de bouc ou de bélier (illustration 7).

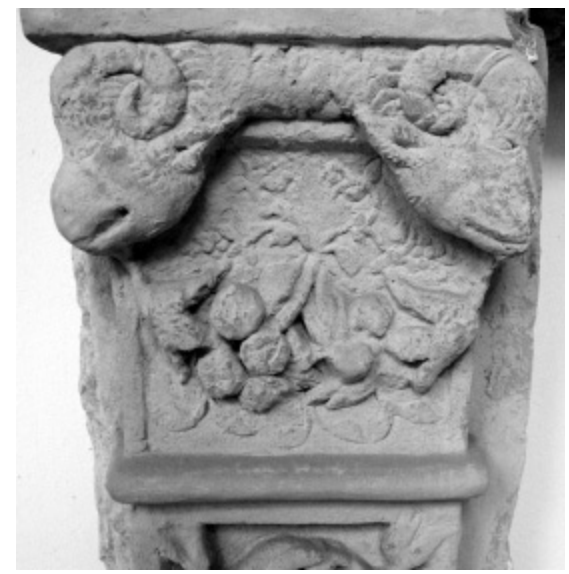

illustration 7 : Anonyme, cheminée du palais épiscopal de Ljubljana, détail, c. 1512 source : photo D. Krašovec

- En dessous, les pilastres ont en leur centre un masque masculin qui rappelle ceux du théâtre antique, courants sur les grotesques (illustration 8).

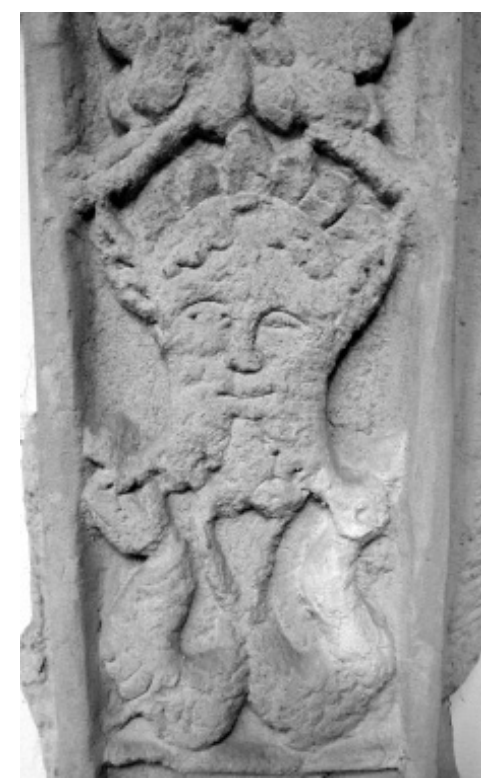

illustration 8 : Anonyme, cheminée du palais épiscopal de Ljubljana, détail, c. 1512 source : photo D. Krašovec 
- Enfin, dans le coin gauche intérieur de la cheminée, il y a un être ailé à serres ou griffes (illustration 9). A priori, rien en nous permet de situer ces êtres dans une quelconque sphère culturelle. Si on prend le dernier, remarquons qu'il ressemble à une sculpture romane de Seckau (illustration 10), donc vue par Raubar et Prygl.

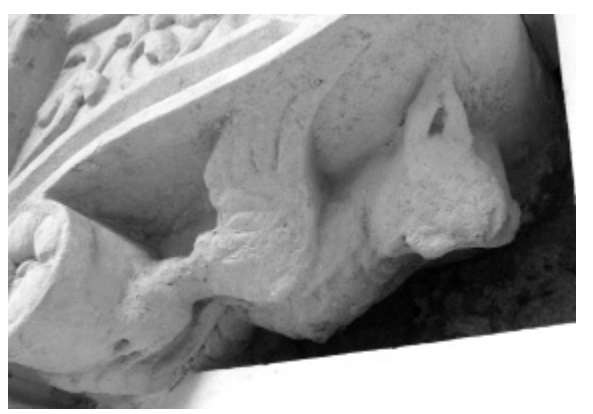

illustration 9 : Anonyme, cheminée du palais épiscopal de Ljubljana, détail, c. 1512 source : photo D. Krašovec

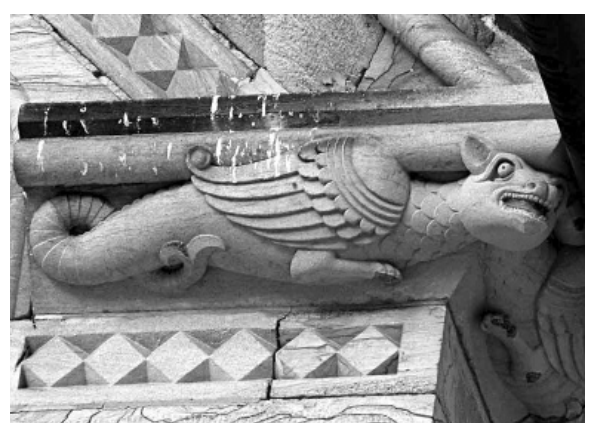

illustration 10 : Anonyme, être hybride sur l'église conventuelle de Seckau, art roman source : http://www.burgenseite.com

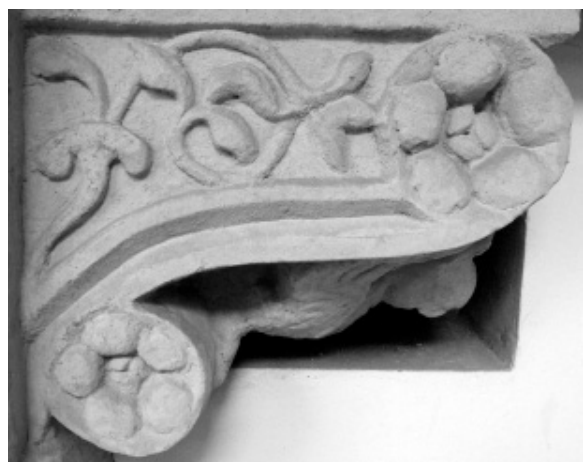

illustration 11 : Anonyme, cheminée du palais épiscopal de Ljubljana, détail, c. 1512 source : photo D. Krašovec 
- Cet être s'appuie sur une fleur à cinq pétales (illustration 11 ; comme beaucoup de fleurs dans la nature), omniprésente dans l'Antiquité romaine (il n'y a absolument aucun doute que Prygl en a vu souvent, par exemple à Aquilée ; illustration 12), mais on la trouve aussi souvent sur les clefs de voûte médiévales, comme celle de Marchegg en Basse-Autriche, vers 1320 (illustration 13). Nous choisissons cet exemple pour les autres motifs floraux, ce motif était très fréquent en Europe.

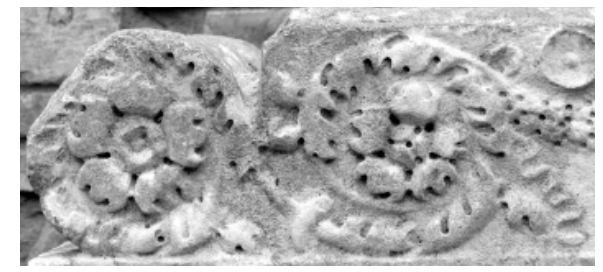

illustration 12 : Anonyme, relief romain, Aquilée source : photo D. Krašovec

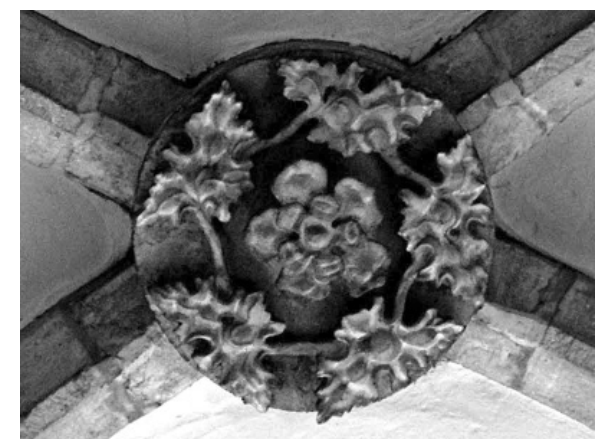

illustration 13 : Anonyme, clef de voûte dans léglise de Marchegg, c. 1320 source : http://www.burgenseite.com

Le procédé de l'hybridation est un phénomène répandu, il est par exemple utilisé à proximité sur le plafond en bois peint de l'église Sainte-Marguerite à Mallestig (illustration 14), en Carinthie, pratiquement à la même date, vers 1510 (Golob, 1988, $50,151)$. Est-ce vraiment une hybridation anthropomorphe ou un homme difforme? Il est presque impossible d'y répondre, l'hybridation étant un phénomène complexe qui fait appel à de nombreux procédés et structures mentales et intellectuelles. A Mallestig, on sent de plus quon touche à la culture populaire et la culture orale. Nous navons plus accès à ces documents. Pour la cheminée, faut-il vraiment chercher des gravures ou des documents imprimés pour chercher une "origine" ? Est-ce légitime ? En quoi une gravure ressemblante est plus pertinente qu'un motif géographiquement plus proche bien que moins ressemblant ? Il est bien connu que les commanditaires - souvent plus que les artistes - recherchent dans les gravures des référents symboliques plus 
quartistiques. Tandis que l'artisan cherche un soutien graphique qui ne correspond pas forcément à son intention créative. A plusieurs niveaux, le référent graphique est donc plus une solution de facilité qu'une intention.

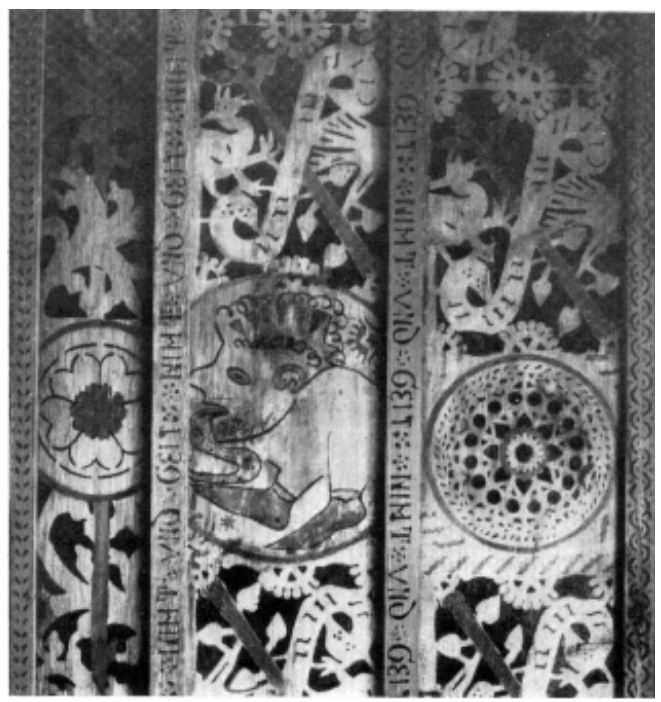

illustration 14 : Anonyme, plafond en bois peint de l'église Sainte-Marguerite, c. 1510, Mallestig source : Golob, 1988, 48

Il ne faut donc pas négliger la dimension de l'oralité dans l'échange entre l'artiste et le commanditaire, d'autant plus que l'oralité faisait partie intégrante de l'écriture, dans sa dimension rhétorique. N’oublions pas que la rhétorique était une matière fondamentale dans l'enseignement des humanistes! Il ne s'agissait pas seulement de bien écrire mais aussi de bien parler.

Surtout, l'hybridation en elle-même ne renvoie à aucune sphère culturelle en particulier. Vers 1500, évoquons pêle-mêle l’œuvre de Jérôme Bosch (c. 1453 - c. 1516), sur lequel on a beaucoup fantasmé de façon injustifiée, de Léonard de Vinci (1452-1519) avec ses caricatures et ses anatomies, celles inspirées des Métamorphoses d'Ovide ou de L'Ovide moralisé du début du XIV ${ }^{\mathrm{e}}$ siècle. ${ }^{19}$ Le corps, beau ou difforme, est un champ de recherche constant, le fantastique "réel/naturel" s'accumule dans les cabinets de curiosités, la corporalité s'exprime jusque dans les contorsions des sectes slovènes quelques années plus tard.

19 Cette version en français est alors plus populaire que l'original, mais dans une aire géographique restreinte, qui ne nous concerne pas ; cf. Possamaï-Pérez, 2009. La version dérivée, Ovidius moralizatus, en prose et en latin vers 1340, quinzième livre du Reductorium morale de Pierre Bersuire, ou Petrus Berchorius, était accessible aux locuteurs non francophones. 


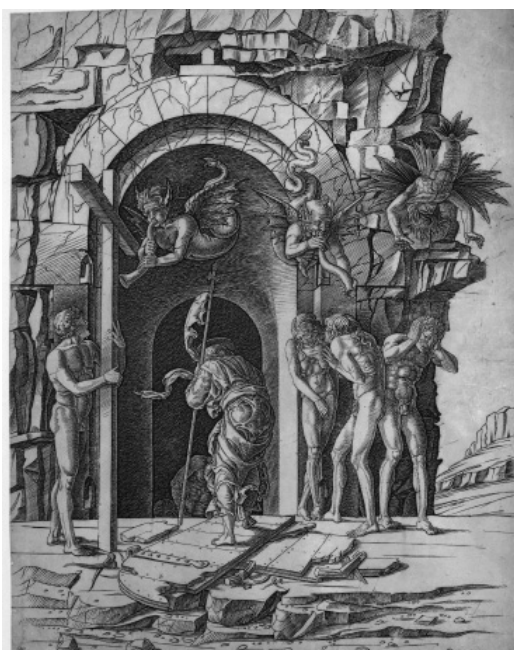

illustration 15 : Cercle d'Andrea Mantegna, Descente du Christ aux enfers, c. 1475-1480 source : http://marinni.livejournal.com/535645.html

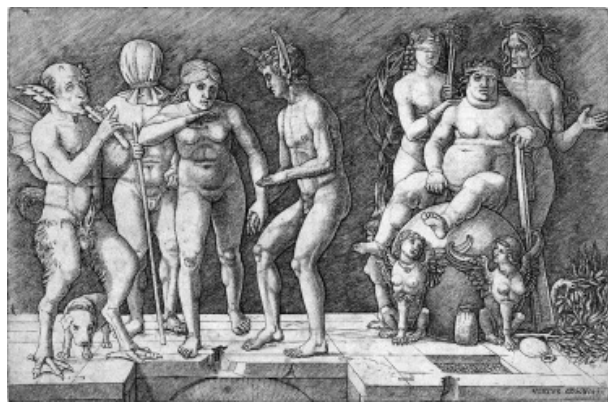

illustration 16 : Cercle d’Andrea Mantegna, Allégorie de la Vertue et du Vice, c. 1490 source : http://marinni.livejournal.com/535645.html

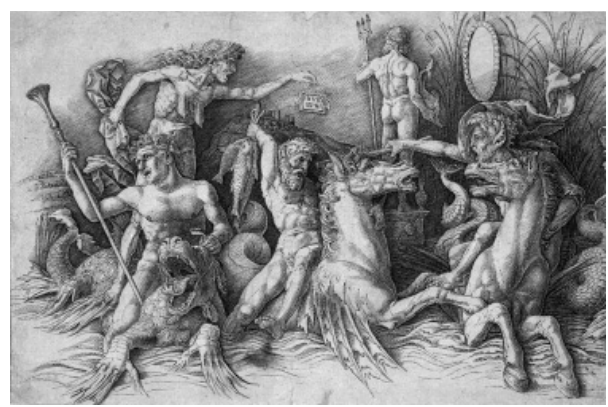

illustration 17 : Andrea Mantegna, Bataille des dieux marins, c. 1470-1480 source : http://marinni.livejournal.com/535645.html 
Mantegna néchappa pas à cette fascination, nous ne pouvons pas le passer sous silence pour plusieurs raisons : il travaille à Padoue où étudient pratiquement tous les intellectuels des provinces méridionales de l'Autriche et il est l'un des premiers grands maîtres à s'investir dans la gravure (on a d'ailleurs songé à sa paternité pour plusieurs illustrations du texte de Colonna). Dans l'ensemble, Mantegna et la cheminée de Ljubljana nont que peu en commun, mais beaucoup de détails se répondent - et c'est ce qui compte pour nous (illustration 15, 16, 17).

Multiplier les points de rapprochement de Bosch à Mantegna signifie qu'on peut, vers 1500, s'inspirer aussi bien de ceux-là que d'une multitude d'autres sans marquer son appartenance à une sphère culturelle précise, on peut librement s'en inspirer pour définir sa propre culture.

Quant aux ornements végétaux décoratifs, il est vain d'en chercher une quelconque origine. Il suffit dobserver la production artistique médiévale pour trouver ces motifs un peu partout, de manière ininterrompue depuis l'Antiquité jusqu'en 1512 (Racinet, A., Dupont-Auberville, M., 2009). Prygl en a consigné quelques exemples dans ses manuscrits (illustration 1). Pour en rester à Mantegna, presque n'importe quel intellectuel de Ljubljana a vu ses œuvres à Padoue, ses décors architectoniques et ses étoffes en trompe-l'œil sont couverts de ces ornements. Or, il est impossible de faire un lien entre ces ornements-là et ceux de la cheminée de Ljubljana. Lagencement est nouveau, mais les ornements végétaux peuvent être trouvés n'importe où, nul besoin de Padoue, de vieux chapiteaux médiévaux autrichiens sont tout aussi riches. Tout au plus, sur les chapiteaux à têtes de boucs, on peut faire le rapprochement entre les végétaux et les attributs de l'abondance.

On prétend souvent que la modes des grotesques prend son essor avec l’œuvre de Raphaël (1483-1520), par exemple avec les décors du Vatican ou de la villa Farnesina à Rome, mais Raphaël ne fait que reprendre un engouement antérieur, conséquence de la découverte de "grottes" archéologiques qui révèlent des décors de l'Antiquité romaine. Surtout, notre cheminée est antérieure aux décors de Raphaël ! Il est encore trop tôt pour vraiment parler de grotesques et le motif du rinceau est prisé depuis longtemps. La question est encore plus compliquée si l’on songe que des Flamands passent maîtres dans l'art de composer ces décors en Italie. Et si notre cheminée est - encore - d'une exécution maladroite, les pilastres décorés de l'épitaphe d'une bourgeoise à St. Veit an der Glan en Carinthie, vers 1520, sont d'une qualité surprenante (illustration 18). C’est-à-dire que la cheminée de Ljubljana apparaît juste avant la diffusion rapide et efficace de ces motifs. Enfin, ces ornements ne connaissent une vraie diffusion par de nombreux ouvrages imprimés que dans les années 1540. Alors que les grotesques ont souvent été interprétés comme une mode décorative importée d'Italie et qui s'acclimate 
très bien chez les “Germains”, il nous semble qu'il faille reconsidérer cette position et mieux prendre en compte le substrat local.

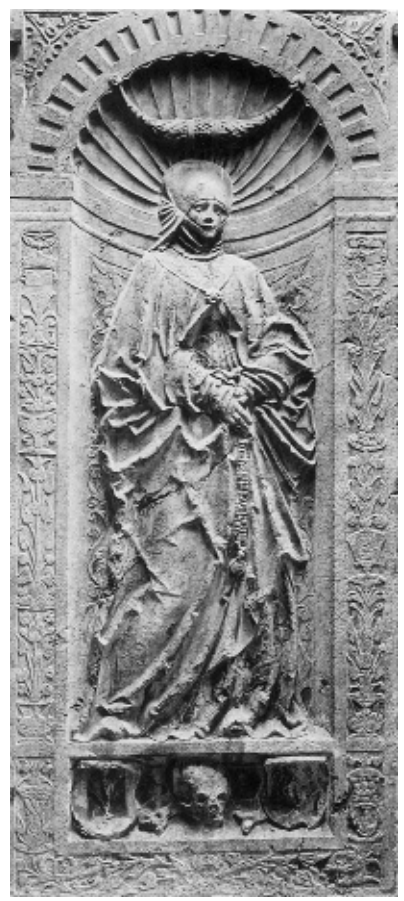

illustration 18 : Anonyme, épitaphe d'une bourgeoise à St. Veit an der Glan, église paroissiale, c. 1520

source : B. Kienzl, W. Deuer, Renaissance in Kärnten, Klagenfurt, 1996

\section{Problématique de la langue}

Cet aperçu montre que nous pouvons jeter des ponts entre différentes sphères culturelles, ce qui enrichit l'œuvre, nous demeurons néanmoins dans l'environnement complexe de cette œuvre sans aller dans aucune direction précise. Cependant, "faire le ménage" dans les liens que nous avons tissés serait injustifié.

Pour cela, il faudrait mieux connaître le contexte. Plutôt que de décrire un contexte historique et intellectuel - c'est-à-dire placer des données précises dans un exposé général - il faut comprendre les enjeux et les failles de l'époque pour libérer l’œuvre des idées préconçues. C'est pourquoi, dans un premier temps, nous cherchons à cerner deux problématiques, celle de la langue et celle du registre, plus particulièrement du registre de langage. Car nous n'avons pas encore soulevé la question de l'agencement des motifs de cette cheminée. 
L'œuvre elle-même est muette. Nous savons uniquement qu'elle est à Ljubljana dans le palais épiscopal dont la construction débute en 1511, on peut raisonnablement dater l'œuvre vers 1512 ou très peu d'années plus tard.

Si l'œuvre est muette, que parlait-on alors à Ljubljana ? Question apparemment simple mais extrêmement complexe ! On y entendait des variantes de l'allemand, du slovène et de l'italien. C'était des langues vernaculaires encore non fixées, il ne faut donc surtout pas les confondre avec les langues qui ont été forgées plus tard. On pourrait croire que seules deux langues sont fixées par une grammaire, le latin et le grec, or ce sont deux langues mortes ! Néanmoins, le grec n'est pas encore accepté par tous en 1512, certains le voient comme un signe d'hérésie ; et le latin est déchiré entre la tradition scolastique et le latin de Térence. En fait, aucune langue n'est encore fixée vers 1512 et avant même de définir des normes, ce qu'on fait quelques décennies plus tard, on se demande encore quelle langue employer. Cela est lourd de sens pour les intellectuels slovènes, car ce choix défini l'appartenance à des sphères culturelles historiques et politiques. Ainsi, l'importance de la langue est aussi patente en politique et dans la diplomatie. Quelle langue doit-on parler si l'on veut gouverner ? Le jeune Maximilien a appris sept langues (Simoniti, 1979, 149154) ! Son précepteur, Prelokar, était slovène. Et les Slovènes étaient des diplomates recherchés pour leurs compétences linguistiques. Maximilien songea à envoyer Raubar en Moscovie, finalement c'est Sigismund Herberstein qui s'y rend. Au retour, il rédige son célèbre récit sur le duché russe en allemand, le texte est mauvais car sa langue maternelle est le slovène, il le fait traduire en latin. ${ }^{20}$ Cependant, pour les Slaves, choisir une langue c'est aussi s'inscrire dans l'histoire : certains intellectuels slaves, dans leur quête des origines mythologiques qui légitime toute dynastie ou nation, essaient alors de retrouver des preuves écrites de leur existence dans l'empire d'Alexandre le Grand. ${ }^{21}$ Cela prouverait non seulement le caractère autochtone des Slaves, au même titre que les Germains et les Romains, mais il donnerait enfin des lettres de noblesse "antiques” à ce peuple qui n’est jamais évoqué par les Anciens. En pointillés on comprend que leur Antiquité est à la fois celle de la culture classique romaine et celle de Jésus, il n'y a pas d'opposition entre paganisme et christianisme. Ce "syncrétisme" perspectif de l'histoire concilie - ou réconcilie ? - différentes aires culturelles et différents pouvoirs, la Rome papale et le Saint Empire romain germanique. ${ }^{22}$

20 Klement de Dobrepolje l'aide dans cette tâche ; sur la relation entre ces deux personnages (Simoniti, 1979, 76-177, 213-215).

21 L'empereur aurait accordé un privilège aux Slaves, nous savons depuis que ce texte est un faux, évoqué à Prague en 1541 par Vaclav Hajek, mais aussi évoqué en 1532 par Vinko Pribojević, de l'île de Hvar, qui fait publier la traduction latine à Venise d'après l'original grec qui aurait été retrouvé à Constantinople (Simoniti, 1979, p. 176-177, 218-219).

22 Nous argumentons et développons ce point de vue dans Krašovec (1500-1520, 2007). 
Les recherches épigraphiques de Prygl peuvent être vues dans cette perspective, inscrire son territoire dans l'Antiquité. Cela ne signifie aucunement être assujetti au référent italien, Raubar est élevé au titre de maréchal dans la guerre contre les Etats de la péninsule italienne ! Cela est bien affirmé sur la plaque inaugurative du palais épiscopal de Ljubljana. ${ }^{23}$ Si les Slovènes choisissent le latin de Térence, c'est qu'ils sont sensibles aux langues vernaculaires, à l'instar d'un Ange Politien qui était doué tant en latin, qu'en grec, qu'en langue... vulgaire. Politien est connu pour un autre précepte où l'homme de culture n'est pas qu'un simple imitateur mais doit s'inspirer de plusieurs auteurs et ne pas perdre son individualité. ${ }^{24}$ A la cour de Raubar on devait connaître cette position et cela se ressent sur la cheminée. D’autant plus qu’elle est encore affirmée par Francesco Colonna, on ne saurait mieux le dire que le chercheur Frédéric Nau :

L'Antiquité est chargée de fournir un ensemble de modèles, mais elle ne doit aucunement être considérée comme une entrave à l'exercice de la liberté et de l'intelligence artistiques, qu'elle est chargée d’encourager au contraire (Nau, $2007,8)$.

Enfin, on aurait tort de croire que toute l'attention était tournée vers l'Italie. Il y avait aussi au sud une fascination pour le Nord, à titre d'exemple rappelons seulement le mot du Florentin Bartolomeo Facio, qui qualifie Jan van Eyck de "principe dei pittori del nostro secolo $»{ }^{25}$ Et gardons-nous aussi de croire que les "grands" artistes italiens étaient ceux que nous avons désignés aujourd'hui, un Pisanello (c. 1395-c. 1455) ou un Francesco Squarcione (c. 1397-1468) étaient plus estimés et considérés comme plus proches de l'antique qu'un Masaccio (1401-1428) alors resté incompris. Si nous voulons décider qui a fondé un nouveau langage artistique, nous devons y réfléchir à deux fois. Un Masaccio était trop radical dans sa rupture tandis qu'un Pisanello intégrait des traditions plus larges. A Ljubljana, au moment où un nouveau langage artistique se forme, on ne recherche pas la rupture, on ne cherche pas à copier des Italiens, on veut assimiler toutes les traditions dont on peut se réclamer.

\section{De la typologie aux lexèmes et aux paradigmes: création d'un nouveau langage artistique}

Diverses problématiques étant posées, concentrons-nous sur la cheminée ellemême. C'est avant tout une construction d'intérieur fonctionnelle, centrale dans les pièces chauffées. Il ne s'agit donc pas d'un objet artistique à proprement parler,

23 Citons l'extrait «RAVBAR (...) ADMINISTR. SACR. RO. IMP. CAES. DIVI. MAXIMILIANI. SVPREMVS. BELLI. VENETI. COMMISSAR. (...)».

24 Pour un contexte élargi et la diffusion de certaines idées d'Ange Politien, cf. André, 2007 ; Laimé, 2007.

25 «Prince des peintres de notre siècle » in De viris illustribus, manuscrit composé entre 1455 et 1457 et publié à Florence en 1745. 
mais d'un support potentiel qui transforme alors le manteau de cheminée en élément décoratif. Endroit chaleureux, central, captant l'attention, il est naturellement destiné à accueillir les attributs - héraldiques et/ou symboliques - du propriétaire des lieux. Objet en trois dimensions, réduit à deux dimensions par le point de vue du spectateur, exposé à de fortes chaleurs et aux fumées, c'est le relief en pierre qui est tout désigné pour l'habiller. Or, une cheminée peut très bien rester nue et ce n'est que vers le XIV siècle qu'on utilise des formes architectoniques pour les montants (généralement la colonne), le linteau (parfois agrémenté d'une corniche) et la hotte ou le trumeau supportant un relief plus ou moins riche. Dans la seconde moitié du XVI siècle, il y a un réel engouement pour les décors de cheminée dans les palais les plus prestigieux d'Europe, on utilise les marbres de couleur, les stucs et la fresque. Vers 1500, on est dans une période intermédiaire, presque de flottement, même les plus belles cheminées d'Italie sont des commandes rares.

Une rupture intervient vers 1460, le célèbre Federico de Montefeltro (1422-1482) commande une magnifique cheminée pour le palais ducal d'Urbino, à l'occasion de son mariage avec Battista Sforza (illustration 19). Les montants sont deux caryatides nues, un homme et une femme qui symbolisent les époux, ce traitement est courageux en 1460. Une frise représente une fête nuptiale païenne et antique, avec danse et musique, des centaures musiciens font partie du cortège. La double frise sur le linteau est inhabituelle, par ses proportions épaisses elle répond au relief médiéval du trumeau. ${ }^{26}$

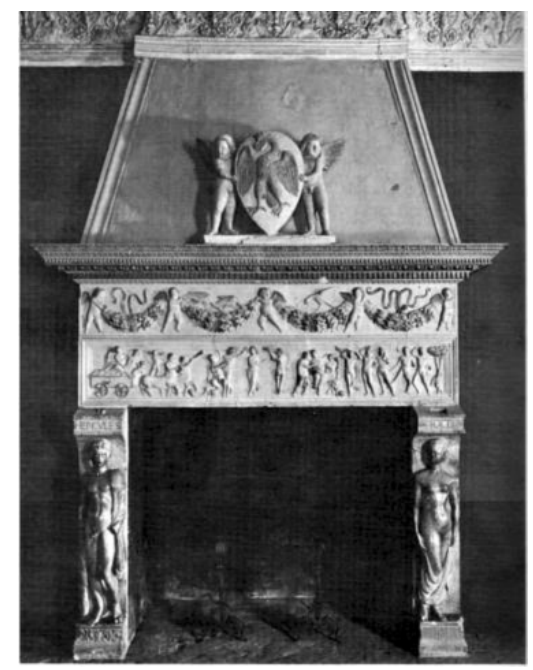

illustration 19 : Anonyme, cheminée du palais d'Urbino, c. 1460 source : inconnue, communication de M. Petit

26 Nous songeons ici à deux exemples géographiquement éloignés, les cheminées du château du duc de Berry à Poitiers, vers 1400, et de l'Hôtel Jacques Cœur à Bourges, vers 1445-1450. 
Non loin de Ljubljana, à la même période, l’art du décor de cheminée prend son essor en Vénétie, dans un style très riche dit gotico fiorito. ${ }^{27}$ Une telle cheminée existe à Ljubljana, elle a probablement été amenée beaucoup plus tard. ${ }^{28}$ Ce qui frappe le plus, c'est la virtuosité, telle cette cheminée récemment mise en vente (illustration 20$){ }^{29}$

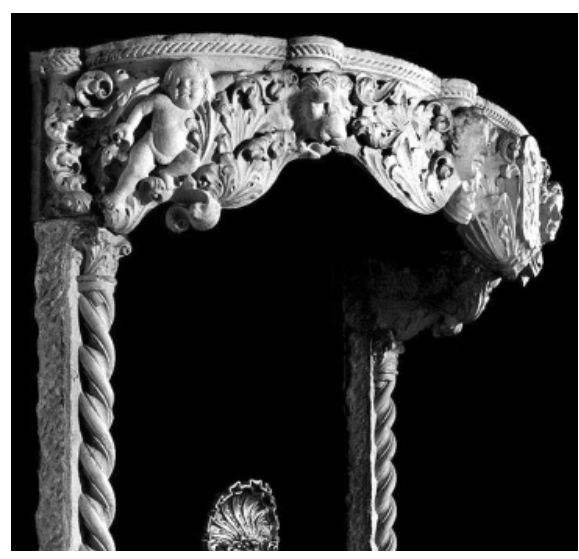

illustration 20 : Jurij de Dalmatie (attr.), cheminée, c. 1450-1473, localisation inconnue source : www.glenrich.ru/218/

Avons-nous pour autant réussi à situer la cheminée de l'évêque Raubar ? Les différences sautent aux yeux, mais pour en rester à la typologie, on pourrait rapprocher la structure élémentaire de deux montants et d'un linteau aux autres exemples. Trois éléments la distinguent : la proéminence de la corniche, de type ionique ou composite à Urbino, de type "Renaissance" ${ }^{30}$ avec frise fleuronnée à Ljubljana ; les chapiteaux sont surmontés d'un élément carré, métonymie d’un architrave au-dessus d'une colonne saillante, élément presque incongru dans les canons des styles; dans l'angle de l'architrave tronqué et du linteau, il y a une console à volute avec un être hybride. Pour ce qui est du "carré" qui surmonte les chapiteaux (il n'existe pas de terme spécifique pour cet élément ainsi isolé), on le retrouve de façon approximative soit dans l'architecture antique (par exemple à l'intérieur du panthéon à Rome) soit dans l'architecture antiquisante romane (par exemple à Saint-Restitut dans le Sud de la France au XII ${ }^{\mathrm{e}}$ siècle). La console, en raison du volatile hybride, fait plutôt songer à la même époque médiévale.

27 Evidemment, nous n'assumons pas ce terme.

28 Elle a été retrouvée dans un immeuble du XIX siècle, rasé en 2006 (Štefanac, 2007).

29 Attribuée à Jurij de Dalmatie, visible sans attribution sur le site www.glenrich.ru/218/ (Štefanac, 2007, 4-5).

30 Certains ouvrages utilisent ce terme pour qualifier des combinaisons ornementales "antiquisantes" mais qui n'étaient pas employées dans l'Antiquité. 


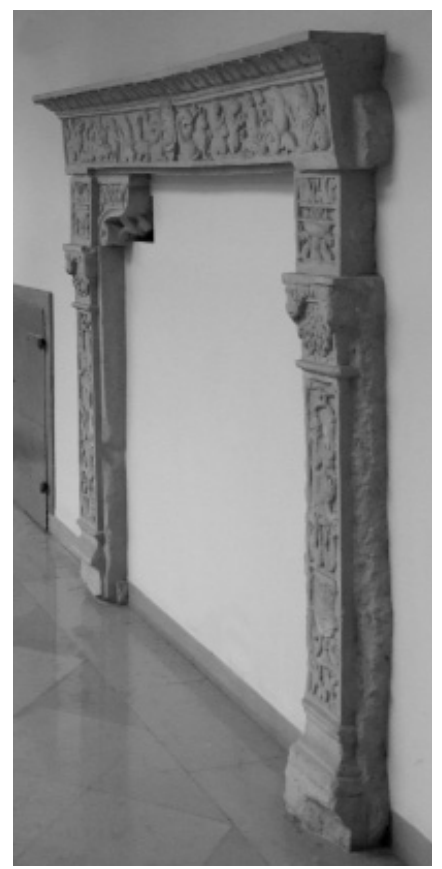

illustration 21 : Anonyme, cheminée du palais épiscopal de Ljubljana, c. 1512 source : photo D. Krašovec

Le plus surprenant de cette typologie, c'est qu'elle ne correspond à aucune géographie, elle interpelle tant l'Antiquité que le Moyen-âge et elle est novatrice - quelle que soit la qualité d'exécution. Ces simples observations, a priori peu originales, sont essentielles : les référents que nous pouvons invoquer sont très vagues géographiquement et temporellement et, surtout, il y a la volonté claire d’être distinct de ses contemporains. On ne fait pas "comme" mais "autre chose".

Pour mieux sonder cette dimension, et faire parler cette œuvre muette, il faut faire appel aux outils propres aux langages. L'usage veut que l'on parle du "langage de l'art" ou de la "grammaire des formes" sans que cela ne soit théorisé en profondeur, on est démuni si l'on veut faire converger différents champs de recherche. ${ }^{31}$ Il y a certes la sémiologie, mais cette science offre de multiples visages, s'applique souvent au cas par cas et investit rarement les $\mathrm{XV}^{\mathrm{e}}$ et $\mathrm{XVI}^{\mathrm{e}}$ siècles. ${ }^{32}$

Si l'on veut bien voir cette cheminée comme un texte littéraire, c'est-à-dire comme un discours, on est vite à court de raisonnements. La dimension du récit

31 Avant toute chose, il faudrait commencer par une clarification des origines de ces expressions.

32 Une exception notable est Marin, 1977 ; il se concentre sur le XVII ${ }^{\mathrm{e}}$ siècle mais fait des incursions dans le siècle antérieur. 
ou du discours est peu claire car on peine à identifier clairement les protagonistes de la frise, le reste ne semble être que décor. Même le rapprochement entre cette frise et la fonction de la cheminée est malaisé à établir : les musiciens renvoient à la fête, les animaux à certains mets, les chapiteaux peut-être à l'abondance, mais cela reste bien faible... Trop d'éléments sur les intentions nous échappent. Il est dès lors pratiquement impossible d'en dégager une lecture intertextuelle qui serait révélatrice de l'auteur, du dédicataire/commanditaire, du lecteur/spectateur. C'est probablement de manière involontaire - en raison de la maladresse d'exécution - qu'il y a dénégation de lénonciation, par ce dernier terme nous entendons cette dimension du discours qui doit entrer en interaction avec le destinataire ; car, outre le discours lui-même, il y a aussi le jeu des regards, la perspective, la gestuelle, qui indiquent des directions et orientent le discours.

Ensuite, au niveau de l'ensemble, non seulement les éléments architectoniques sont faiblement liés entre eux, ils sont juste superposés, mais ils ne correspondent pas aux canons du "langage architectural". Après la redécouverte de Vitruve, et les écrits d'Alberti, la superposition des ordres était d'une brûlante actualité, ici il n’y en a aucun écho alors que le simple motif de rinceaux et de "grotesques" sur des pilastres est un écho de lépoque. Peut-on pourtant attendre des hommes de Ljubljana qu'ils soient solidaires de ces discussions? Car, comme le note Colonna, « les illustrations perdues (de Vitruve) font l'objet de débats » (Nau, 2007, 4), et donc pourquoi l'entourage de Raubar devrait accepter tous les modèles contemporains ou antiques qui sont à leur disposition?

Ainsi, malgréles apparences, on està mille lieues des débats architecturaux d'actualité dans la péninsule italienne, et les chapiteaux à têtes de boucs ne renvoient à aucun ordre connu. Deux remarques s'imposent : l'une, c'est que Tyfernus est aussi l'auteur de constructions "gothiques", l'autre c'est que l'architecture de l'Autriche intérieure est très imaginative en 1500-1520, il y a une véritable "euphorie des formes" (Krašovec, 2007, 149-155). Cette cheminée s'intègre parfaitement à ce cadre où les forces créatrices qui travaillent les formes sont à l'œuvre dans différentes régions européennes, leur confère une unité globale, malgré l'apparent hétéroclisme des résultats. Tant qu’on voudra voir une opposition entre "gothique flamboyant" et "Renaissance", on naura pas compris cette époque. Colonna est plus subtil, certaines de ses descriptions empruntent tant au merveilleux médiéval quà l'Antiquité, et il va même jusqu'à convoquer Pline l'Ancien dans son récit pour qu'il soit témoin de la supériorité des constructions de la fin du XV siècle sur celles de l'Antiquité (Nau, 2007, 5, 7).

Il faut considérer cette cheminée en dehors de tout système référentiel centré sur l'Italie, ou une autre région, cette œuvre se définit elle-même comme le propre centre de son système référentiel, et l'affirme par ce moyen. Si différentes orientations 
esthétiques, ou régions, puisent à quelques sources communes, cela ne définit aucune communauté artistique et n'est révélateur d'aucune appropriation directe, ni du "copieur" sur le "copié" (appropriation symbolique), ni du "copié" sur le "copieur" (“complexe de supériorité"33).

Les artistes et commanditaires de Ljubljana ont donc voulu créer quelque chose de nouveau en puisant à l'intérieur d'une aire culturelle dont l'horizon est très vaste. Penchons-nous alors sur la "structure linguistique" pour mieux cerner ce "langage artistique". Avant même de s'intéresser aux signes ou aux lexèmes, observons la tension entre la surface/support et ce qui est représenté. Cevc utilise l'expression d'horror vacui (Cevc, 1981, 26), d'utilisation courante, il faut néanmoins remarquer que ce terme est popularisé par Aristote quand il l'applique à la nature. Vers 1500, cette expression est effectivement utilisée, mais en physique, pas en art. Dans ce dernier champ, elle n'apparaît qu'avec l'historien d'art italien Mario Praz (1896-1982) pour décrire l'atmosphère étouffante de l'Angleterre victorienne.

L'horror vacui, si on ne veut pas en faire juste une expression pour décrire "du remplissage", suppose une organisation consciente pour combler et aménager la surface ou un volume susceptibles d’être abandonnés au vide. Dans notre cas, il y a absence de vide. Ce n'est pas qu'un jeu rhétorique, ce qui nous fait dire cela c'est justement l'absence d'une rhétorique apparente et évidente. Le décor est couvrant et n'est pas en interaction avec son support ; tandis quà Urbino la surface de l'arrière-fond est très présente en créant un espace de tension, et que dans les modèles vénitiens on passe à l'extrême inverse, il n'y a plus du tout de surface, sa négation est radicale. Cette dimension ne doit pas être sous-estimée, elle était l'un des pôles de la réflexion en histoire de l'art vers 1880-1910 ; Claude Zilberberg revisite les textes d'Alois Riegl (si important pour l'historiographie slovène, il a formé plusieurs des premiers professeurs de Ljubljana) et de ses contemporains, il explore les aires de l'accent et de l'in-accent, les aires contrastives ou corrélatives. ${ }^{34}$ A Ljubljana, la surface n'est pas un paradigme (dans une perspective d'énonciation), cela lui enlève déjà toute possibilité dêtre en interaction avec les autres paradigmes et leurs systèmes de désinences. Il y a en fait une crainte du vide car il isolerait les éléments éloignés, alors que le vide doit être l’occasion de mettre en valeur d’autres interactions, entre ce qui est représenté, ou ce qui ne l’est pas.

Ce sont ces systèmes que nous allons observer en commençant par la frise. Faute de pouvoir nommer les êtres animés - et dans l'impossibilité même de nommer des catégories générales - on peut seulement ajouter que parmi les animaux que nous

33 Nous ne pensons pas au concept de psychologie qui masque un complexe d'infériorité, mais au sentiment de valorisation indue qui ne comprend pas la démarche de ceux qui prolongent une lignée dont on n'est qu'un maillon et non l'origine.

34 Zilberberg, 2007 ; cette étude est d'un apport restreint dans notre cas précis, mais elle peut apporter beaucoup si on l'applique à d'autres objets artistiques de cette époque dans la même région. 
reconnaissons certains sont domestiques d'autres sauvages. Or, déjà là, tout se brouille à nouveau! Lours dressé sur ses pattes arrière, est-il sauvage ou domestique ? Quel est le "degré de civilisation" du bovin, est-ce un bœuf castré et apprivoisé ou un taureau encore dans son état "sauvage" primitif? Dans l'impossibilité de nommer des catégories générales (naturelles, morphologiques ou temporelles/historiques pour ce qui relève du référentiel), considérons chacun de ces êtres comme un syntagme. A regarder les "musiciens" de plus près, il ne s'agit pas d'“hybrides intégrés" mais de parties de corps juxtaposées - tandis qu’à Urbino, ou chez Mantegna, nous avons de véritables centaures aux corps harmonieux. A Ljubljana, il ne s'agit pas de paradigme à proprement parlé mais plutôt de syntagme composé d'au moins deux morphèmes lexicaux. Et si, du coup, on avait affaire à des êtres doubles, c'est-à-dire que ces "musiciens" sont des protagonistes “déguisés" pour une fête?

En revanche, tous les autres êtres vivants de la frise sont des syntagmes élémentaires. D’un point de vue paradigmatique, quels sont les lexèmes qui créent des relations grammaticales, a-t-on un système de désinences ? De genre ? De nombre ? Il y a un embryon de nombre, certaines figures apparaissent deux fois, les autres une seule, nous avons donc le singulier et le duel. ${ }^{35}$ Or le duel répond à la logique de la symétrie, c'est plus un duel compositionnel que grammatical, il serait donc aventureux d'invoquer le pluriel - cela semblerait même exclu car il brise la symétrie. ${ }^{36}$ Pour ce qui est du genre, nous avons deux êtres anthropomorphes féminins, tous les autres sont des êtres masculins ou ne peuvent être définis, ils sont donc neutres. Plus intéressant, les figures féminines sont isolées tandis que celles qui sont en interaction sont masculines ou neutres $:{ }^{37}$ les phrases, ou groupes énonciatifs autonomes, ignorent le genre féminin. Hasard ou pudeur dans un archevêché ? Dans toute l'Autriche intérieure, le nu féminin est banni, il ne fait qu'une apparition furtive dans les années 1560 (Krašovec, Culture, 2007, 405-407), les mentalités sont patriarcales, cette “omission" est en fait révélatrice. A Urbino, on n’a laissé aucune place à la pudibonderie.

Quelles sont ces “phrases”, ces groupes où on peut observer une cohésion sémantique? Il y en a deux, autour des "musiciens". Les regards et les gestes sont des lexèmes qui marquent des rapports entre les paradigmes, tout converge vers les musiciens : ils sont donc le sujet d'une phrase, leur instrument de musique agit comme un verbe transitif. Les autres protagonistes sont soit des compléments, soit des subordonnées mais seule la disposition dans l'espace agit comme connecteur grammatical.

35 Le duel est encore en usage dans le slovène contemporain et se distingue véritablement du pluriel, ce n'est pas une "curiosité" linguistique.

36 Deux unités reproduites par la symétrie conduisent au chiffre quatre, donc un pluriel, mais on ne peut obtenir les chiffres impairs trois, cinq, etc.

37 Les animaux représentés ne sont pas sexués, nous ne parlons pas du genre grammatical des noms communs, différents d'une langue à l'autre. La licorne est au masculin en latin et en slovène, au neutre en allemand. 
Dans ces groupes énonciatifs, il est difficile de distinguer d'autres relations, c'est-àdire des verbes intransitifs, on est dans des interactions a minima. Si l'on appliquait ces outils à la frise d'Urbino, les résultats seraient beaucoup plus riches. Nous avons donc une grammaire bancale, peu complexe, seulement à létat d'ébauche : sachant que les personnes du cercle de Raubar et Prygl sont très cultivées, douées pour la rhétorique, où leurs ambitions ou intentions ont-elles achoppées? Il y a deux endroits où ils ont pu trébucher.

D’abord, dans leur volonté même de créer un langage, ils ont rejeté ceux qui étaient en usage. Pourquoi n’avoir pas tout simplement copié ce qui existait déjà ? On peut donc au moins affirmer qu'ils nétaient pas des "barbares maladroits", fiers d'une culture dont ils ne trouvaient pas d'équivalents ailleurs. Cependant, ils nont pas réussi malgré leur imagination et leur potentiel créateur à obtenir un langage nouveau d'une complexité comparable aux langages existants. Peut-être étaient-ils mal à l'aise avec certaines de leurs sources car, comme le remarque Frédéric Nau, les siècles passés ont rendu l'Antiquité moins familière ; le héros de Colonna, Poliphile, avoue contempler avec « une admiration continuelle, pensant en moi-même qu'elle pouvait être la signification de ces histoires que je trouvais bien obscures... » (Nau, 2007, 11).

Ensuite, en admettant qu'ils aient réussi à créer un nouveau langage complexe, comment auraient-ils transmis ce langage à un artisan médiocre ? Comment est-on passé du commanditaire à l'artiste : en donnant des instructions orales, écrites, ou dessinées ? Quels mots utiliser pour se faire comprendre alors qu'aujourd'hui on est incapable de nommer la plupart des hybrides ou animaux ? Lartisan a-t-il compris ce qu'on lui disait ? Ce qu'on voulait de lui ? Ces questions sont d'autant plus complexes que l'intellectuel vers 1500 ne savait pas y répondre. Les contemporains ont bien sûr identifié la proximité de Colonna, Vitruve et Alberti, par exemple Jean Martin $(\dagger 1553)$ qui a traduit et imprimé les trois auteurs en français avec l'aide de Sebastiano Serlio $:{ }^{38}$ mais chacun doit faire face aux dilemmes de Colonna qui a eu les plus grandes difficultés à concilier les termes grecs, latins et modernes dans les traductions et équivalences (Nau, 2007, 12).

Et comment traduit-on en langage plastique des instructions énonciatives ? Le résultat doit-il être aussi énonciatif ? Ou le simple fait de donner une instruction (ne serait-ce que de dire de copier un modèle) fait changer le discursif de registre (le commanditaire "voit" un récit, l'exécutant voit juste les figures sans le récit) ? La dernière question renvoie à la créativité de l'artiste : doit-il obligatoirement voir les formes et figures comme paradigmes pour créer un nouveau langage artistique, c'est-

38 Respectivement en 1546, 1545-1547 et 1553; Martin a retouché une traduction antérieure et anonyme de Colonna. 
à-dire au moins anticiper un système de désinences avant de pouvoir y intégrer de nouveaux éléments ? ${ }^{39}$

Dans le cas de la cheminée de Ljubljana, ces questions sont sans réponses évidentes faute de mieux connaître le contexte le plus immédiat. Au-delà du problème du langage, nous avons vu qu'il y a aussi le problème du référentiel, il est impossible de dire ici qu'il s'agit d'une œuvre “d'inspiration Renaissance qui fait référence à l'Antiquité". Il y a à l'évidence un mélange de registres, médiéval, antique, voire populaire. On sousestime trop souvent ce dernier registre alors que même Alberti, en relevant les dicta de Pythagore, y ajoute les siennes ou celles issues de la culture populaire, considérant qu'elles pouvaient avoir autant de valeur (Vuilleumier-Laurens, 2000, 25-39).

Dans la relation commanditaire - exécutant - spectateur, il faut aussi se demander si chacun perçoit ces registres de la même façon. Il ne faut pas non plus exclure que ce que nous voyons aujourd'hui est un "compromis", volontaire ou non, graphique et compositionnel - mais sûrement pas iconologique et iconographique. Ici, bien sûr, il ne faut pas oublier le probable manque de savoir-faire théorique de l'artisan, il est trop facile de toujours invoquer sa maladresse technique.

\section{Le rhizome ou l'absence de hiérarchie entre les oeuvres}

Pourquoi sêtre intéressé à cette cheminée plutôt quà une autre œuvre, plus riche, de meilleure qualité, où il aurait été plus facile de jongler avec les termes, où il aurait été plus facile de découvrir des interactions ? Parce que pour les œuvres plus riches il existe déjà de nombreuses méthodes d'analyse tandis que cette cheminée résistait à toutes les tentatives d'analyse, elle restait murée dans le silence des généralités. Il fallait élaborer de nouveaux outils pour la "faire parler" et s'affranchir des généralités qui effacent la richesse d'un contexte particulier.

Pour une conclusion fort simple : il ne s'agit ni de Renaissance, ni de rien entre le gothique et le baroque, c'est tout simplement une œuvre révélatrice du milieu intellectuel vers 1510, génération qui est distincte de la précédente et de la suivante. C'est pour cela quelle n'a pu être crée quà ce moment-là, ni avant, ni après. Il est encore plus erroné d’en déduire que la "Renaissance" n’a pas accroché à Ljubljana, qu’elle est arrivée trop tôt, ou que le milieu nétait pas assez mûr pour l'assimiler. Au contraire, on perçoit une réflexion sur le langage qui annonce les changements ultérieurs : nouvelle grammaire pour le langage des formes, nouvelle perception des langues, prise de conscience de la valeur des langues vernaculaires et de la culture locale.

39 Nous limitons notre questionnement à cette période, vers 1500, de nombreuses études ont abordé ces aspects pour l'art contemporain. 
A toujours vouloir s'en référer à l'Italie, les historiens de l'art s'enlèvent la possibilité de comprendre ce monde. Il est absurde de chercher une "Renaissance italienne" en Carniole, alors qu'on peut trouver de nombreux points communs dans la réflexion - et non dans les réalisations. Dans le cercle de Raubar comme chez Francesco Colonna, l'architecture n'est « ni antique ni moderne : elle est, avant toute chose, imaginaire et mentale » (Nau, 2007, 7 ; c'est lui qui souligne), et donc les reliefs de Ljubljana ou le texte littéraire vénitien " engage(nt) une méditation sur la signification du modèle ancien dans une culture moderne » (Nau, 2007, 14).

Malgré la passion pour l'Antiquité, l'un des principaux écueils à surmonter dans la formidable aventure pour former de nouveaux langages - au sens le plus large - ce sont « les imperfections et les impossibilités de la transmission de la culture ancienne » (Nau, 2007, 21) 40 $^{4}$ et la coupure entre les Anciens et les Modernes, qu'aucune filiation ne peut combler.

Beaucoup de questions posées dans cette étude restent ouvertes et il appartient aux recherches ultérieures de les approfondir. Il y a pourtant un aspect important qui n’a pas été abordé - et qui nous a néanmoins guidé dans notre réflexion : c'est le rôle de l'historiographie contemporaine et de l'apparition de ses concepts au XIX siècle sur notre perception du passé. ${ }^{41}$ Qu'on le veuille ou non, nous prenons pour acquises des méthodes de l'histoire de l'art forgées à cette époque et les utilisons aujourd'hui sans vouloir comprendre leur genèse et sans les rénover.

Pour la plupart des régions européennes dites moins “évoluées”, il faut abandonner l'idée d'influence et appliquer l'idée de rhizome de Gilles Deuleze, et Félix Guattari, pour saisir les interactions entre les réalisations intellectuelles et artistiques, c'està-dire qu'il n'y a pas de subordination hiérarchique, tout élément peut agir sur tout autre quelle que soit sa position (Deleuze, Guattari, 1980). En Carniole, la cheminée archiépiscopale est un nœud très fertile dans un rhizome encore peu exploré.

40 Que l'on songe aussi aux réticences de Trubar pour traduire la Bible en slovène, certains arguments sont dans cet esprit et c'est justement un Italien, Pier Paolo Vergerio, qui l'a convaincu de se lancer dans cette entreprise.

41 Pour se libérer des schémas de l'histoire de l'art slovène, italienne et autrichienne, le lecteur aura constaté que nous avons majoritairement utilisé la littérature scientifique francophone pour nos analyses. Ce n'est pas par commodité linguistique mais en raison d'une réelle nécessité épistémologique. 


\section{Sources}

Francesco Colonna, Hypnerotomachia Poliphili, 1499 (texte original accessible sur Internet).

Laurentius Valla, Elegantiae linguae Latinae (1435-1444; publ. posthume 1471).

Giorgio Vasari, Le Vite de’ piú eccellenti architetti, pittori, et scultori (texte original accessible sur Internet).

\section{Bibliographie}

André, M.-F., La classification des artes et sa dimension critique dans la Praelectio sur Suétone de Nicolas Béraud (1515), in : Camenae 1 : Philosophie, rhétorique et poétique latines, de l'Antiquité à la Renaissance, 2007 (en ligne).

Biard, J., éd., Le Langage mental du Moyen Âge à l'âge classique, Paris 2009.

Cevc, E., Poznogotska plastika na Slovenskem, Ljubljana 1970.

Cevc, E., Kiparstvo na Slovenskem med gotiko in barokom, Ljubljana 1981.

Deleuze, G. et Guattari, F., Capitalisme et Schizophrénie 2 : Mille Plateaux, Paris 1980.

Descoings, K., Barbarus hic ego sum. Le poète étranger de l'élégie d'exil, dans l'Antiquité et à la Renaissance, in : Camenae 1, op. cité, 2007.

Doudet, E., Translatio du même au même : autorité et identité littéraires dans les traductions vernaculaires à la fin du Moyen Âge, in: Camenae 3 : Translations. Pratiques de traduction et transferts de sens à la Renaissance, 2007 (en ligne).

Faidutti, B., Images et connaissance de la licorne (fin du Moyen Age - XIXe siècle), Paris 1996 (http://faidutti.free.fr/licornes/these/these.html).

Febvre, L., Michelet et la Renaissance, Paris 1992.

Golob, N., Poslikani leseni stropi na Slovenskem do sredine 18. stoletja, Ljubljana 1988.

Haslag, J., Gothic im siebzehnten und achtzehnten Jahrhundert, Köln, Graz 1963.

Holbrook, W. C., The Adjective gothique in the $18^{\text {th }}$ century, in : Modern Language Notes 56, 1941, pp. 498-503.

Klima, G., The Medieval Problem of Universals, in : The Stanford Encyclopedia of Philosophy, 2008 (http://plato.stanford.edu/entries/universals-medieval/).

Krašovec, D., 1500-1520 : Redécouvrir la mémoire des slaves d'Autriche dans l'Antiquité ou choisir entre la Renaissance et le gothique flamboyant, in : Mémoires, récit, histoire dans l'Europe des XVI et XVII' siècles, Nancy 2007, p. 141-158.

Krašovec, D., La Culture visuelle en Autriche intérieure à l'époque moderne, thèse de doctorat non publiée, Nancy 2007. 
Laimé, A., L'influence d’Ange Politien dans la préface des Silvae de Nicolas Petit (1522), in : Camenae 1, op. cité, 2007.

List, R., Stift Admont 1074-1974 : Festschrift zur Neunhundertjahrfeier, Graz, 1974.

Marin, L., Détruire la peinture, Paris 1977.

Nau, F., I/Y : la culture de la Rome antique dans l'œuvre de Francesco Colonna (1499), in : Camenae 2 : Roma Aeterna : voir, dire et penser Rome de l'Antiquité à la Renaissance, 2007 (en ligne).

Possamaï-Pérez, M., Nouvelles études sur l'Ovide moralisé, Paris 2009.

Racinet, A., Dupont-Auberville, M., L'Ornement polychrome (1869-1888) et L'Ornement des tissus (1877), rééd. The World of Ornament, Köln 2009.

Simoniti, P., Humanizem na Slovenskem in slovenski humanisti do srede XVI. stoletja, Ljubljana 1979.

Štefanac, S., Kamin iz Šumija - pomembno delo beneškega "gotico fiorito“v Ljubljani, in : Umetnostno kronika 17, 2007, p. 2-5.

Šumi, N., Arhitektura sedemnajstega stoletja na Slovenskem, Ljubljana 1966.

Thénard-Duvivier, F., Hybridation et métamorphoses au seuil des cathédrales, in : Images Re-vues 6, 2009 (http://imagesrevues.revues.org/686).

Vuilleumier-Laurens, F., La Raison des figures symboliques à la Renaissance et à l'âge classique, Genève 2000.

Zilberberg, C., Riegl et l'invention du paradigme, in : Nouveaux actes sémiotiques, 2007 (http://revues.unilim.fr/nas/document.php?id=1482).

Note: la disponibilité de tous les articles en ligne a été vérifiée en septembre 2011. 


\title{
Premišljevanja o govorici nekega reliefa iz leta 1512: ne renesansa ne gotika ali kako preseči meje umetnostne zgodovine
}

\begin{abstract}
Ključne besede: kamin škofijske palače v Ljubljani, renesansa, gotika, hibridizacija, jezik, likovna govorica, umetnost in lingvistika
\end{abstract}

Kamin škofijske palače v Ljubljani je eno od najbolj nenavadnih umetniških del z začetka 16. stoletja. S katerimi konteksti je treba povezati to anonimno delo, ki je nastalo okrog leta 1512? To je bistveno vprašanje, ki ga je treba razrešiti brez posploševanj, kar pomeni, da se je treba soočiti s problematiko, namesto da bi se ji izogibali. Ta kamin, ki so mu raziskovalci namenjali le malo pozornosti, naj bi vseboval prvine "gotike« in »renesanse«, toda te besede leta 1512 niso imele nobenega pomena, preprosto niso obstajale. Edina beseda, ki so jo takrat uporabljali v škofijski palači v Ljubljani, je bila »eleganten«, in to besedo je treba postaviti v nasprotje $\mathrm{z}$ drugim izrazom, ki je bil takrat v Evropi zelo v modi, »barbarski«. Med osebnostmi, ki jih je treba umestiti okrog tega dela, sta škof Krištof Ravbar in humanist Avguštin Prygl, opredeliti pa je mogoče tudi širši intelektualni krog z Bernardom Pergerjem, Lorenzom Vallo, Angelom Polizianom in Francescom Colonnom. Če smo pozorni na spise teh avtorjev, še posebej na Colonnove, ni pravega vrednostnega nasprotja med njihovim časom in antiko. Pri njej se navdihujejo, da bi ustvarili govorico - nosilko kulture -, ne da bi zavračali srednjeveško umetnost, pri čemer so spoštovali superiornost krščanstva.

Težko je poimenovati bitja, upodobljena na kaminu škofijske palače, in poimenovanje je lahko celo nevarno. Ne smemo prezreti tega semantičnega problema, kajti vsako navajanje imena se sklicuje na določeno kulturno ali jezikovno ozadje, ki nezavedno usmerja naše sklepanje. Prav tako je nemogoče uporabljati besedišče tiste dobe, ker pogosto ne vemo, katere jezike so govorili naročniki in umetniki ter kako so prilagajali ali prevajali določene izraze (to je bil pravi intelektualni problem že okrog leta 1500 - spor o nominalizmu, prve teorije prevajanja, Colonnova semantična oklevanja). Zavedali so se tudi, da ime trdno določi neko poljubno resničnost ter ne razreši problema razmerja med jezikovnimi bitnostmi in miselnimi predstavami. Tako zadnje raziskave na temo hibridizacije in metamorfoze zavzamejo stališče, da je treba bitja predvsem dobro opisati in jim ne dajati imen, ker jih lahko s tem iztrgamo iz njihovega konteksta. 
V Ljubljani ne vemo, ali gre za tuja, imaginarna, sanjska ali mitska bitja, preprosto za stare zglede ali igrive stvaritve. Poleg tega je bil proces hibridizacije v tistem času po Evropi zelo razširjen na različnih ravneh kulture, vsak primer je specifičen. Z enakimi težavami se srečujemo pri dekorativnem rastlinskem okrasju, ki ne napotuje na nobeno posebno kulturno sfero - ali na več sfer hkrati.

Da bi bolje razumeli kontekst, bomo skušali zamejiti problematiko govorice umetniške, književne in domače. Ob preučevanju, kako je sestavljen kamin, lahko analiziramo tip govorice, ki je uporabljen. Noben jezik še ni določen (niti latinščina ne) in izbrati neki jezik pomeni tudi, da se človek vpiše v zgodovino, v politično in zemljepisno polje. Ker je Ljubljana križišče, so bile izbire nabite s pomenom, ljudje so se hoteli vpisovati v različna kulturna področja, med različne oblasti, od Rima, prek Svetega rimskega cesarstva in tja do slovanskega sveta. V tem kontekstu je bila naloga antike ponuditi celoto modelov, vendar pa je nikakor ne smemo obravnavati kot oviro za udejanjanje umetniške svobode in inteligence. To dvoje je imela, nasprotno, nalogo spodbujati.

Analiza tipologije kamina kaže, da ne ustreza nobeni geografiji, interpretira tako antiko kakor tudi srednji vek in je inovativna - ne glede na kakovost izdelave. Prisotna je torej jasna volja po razlikovanju od svojih sodobnikov. Da bi bolje preučili to razsežnost, je treba poseči po orodjih, lastnih govoricam. Mogoče je uporabiti splošen izraz "govorica umetnosti«, semiologija ali deli diskurza v retoriki, toda to nam le malo pomaga.

Preveč prvin glede namenov se nam izmika. Zato je praktično nemogoče, da bi se na njihovi osnovi lotili medbesedilnega branja, ki bi razkrilo avtorja, osebo, ki ji je delo posvečeno/naročnika, gledalca. Tudi ni več sledu o razpravah o »arhitekturni govorici«, toda interpretacije Vitruvija so bile še zelo polemične - in torej vir svobode. Dejansko kamin ustreza izjemno nenavadnemu ukvarjanju z oblikami, do katerega je v Avstriji in Evropi prišlo v letih 1500-1520.

Torej je treba kamin škofijske palače v Ljubljani poskusiti gledati in obravnavati zunaj vsakega referenčnega sistema, osredotočenega na Italijo ali na kakšno drugo pokrajino. To delo se opredeljuje samo kot lastno središče svojega referenčnega sistema. Če analiziramo like kot sintagme s sistemom paradigem in leksemov, je mogoče razbrati »jezikovno strukturo«. Najprej, površina ali praznina nimata vrednosti paradigme, če uporabimo teorije Aloisa Riegla in Clauda Zilberberga, liki so torej omejeni na preprost diskurz brez razmerja s kaminom, ki je njihov nosilec. Kar zadeva slovnična obrazila, je sistem števila in spolov zelo poenostavljen, interakcije med različnimi prvinami kamina so torej omejene na najmanjšo možno mero. Sami liki so sestavljeni iz leksikalnih in ne gramatikalnih sintagem, s prehodnimi glagolskimi razmerji. Ta 
poenostavitev govorice, v kateri pa ostaja nekaj jasnih pravil, vendarle osvetljuje napor in premišljevanje obdobja, kako narediti za svojo govorico in kulturo preteklosti, z namenom utrditi in poveličati sedanjost ter ne obuditi v življenje časa, ki so ga takrat že imeli za mrtvega. Na semantični ravni je velik problem, kako na novo izraziti svoje znanje, da bi ga mogli prenesti dalje. Na primer: obrtnik, ki je delal kamin, ni imel jezikoslovnega, kulturnega in teoretičnega znanja, da bi razumel napotila humanistov škofovskega dvora v Ljubljani, in iz tehničnega vidika je njegova izdelava slab prevod namenov naročnikov.

Zakaj je vredno, da se posvetimo kaminu škofijske palače $\mathrm{v}$ Ljubljani in ne kakšnemu drugemu delu, bogatejšemu in bolj kakovostnemu, pri katerem bi se bilo lažje poigravati z izrazi, kjer bi bilo lažje razbirati interakcije? Zato, ker za bogatejša dela že obstajajo številne analitične metode, medtem ko se je ta kamin upiral vsem poskusom analize, ostal je zazidan v molk posploševanj. Treba je izdelati nova orodja, da bi ga pripravili k temu, da »spregovori«, in se osvoboditi splošnosti, ki izbrisujejo bogastvo posamičnega konteksta.

Tako v Ravbarjevem krogu kakor pri Colonni ni umetnost ne antična ne moderna, predvsem je nekaj domišljijskega in duševnega. Treba je opustiti idejo vpliva in uporabiti idejo micelija Gillesa Deleuza in Félixa Guattarija, da bi dojeli medsebojno vplivanje med intelektualnimi in umetniškimi stvaritvami, se pravi, da ni hierarhične podreditve, da lahko vsaka prvina vpliva na vse druge ne glede na njihov položaj. Na Kranjskem je škofijski kamin zelo rodovitno vozlišče v še dokaj neraziskanem miceliju. 